\title{
Improved intranuclear cascade model for nucleon-nucleus interactions
}

\author{
J. Cugnon ${ }^{1}$, C. Volant, S. Vuillier \\ CEA DAPNIA/SPhN, CE Saclay, F-91191 Gif-sur-Yvette Cedex, France
}

Received 18 December 1996

\begin{abstract}
The intranuclear cascade (INC) plus evaporation model for nucleon-nucleus interactions in the $200 \mathrm{MeV}-1.2 \mathrm{GeV}$ range is revisited. The standard Liège INC model supplemented by the Dresner evaporation-fission model is used and shown to give a good overall agreement with experimental data, basically neutron double differential cross-sections and residue mass spectra. Small systematic discrepancies are identified and shown to correspond to the single scattering contribution. Improvements of the INC model, linked with in-medium cross-sections, refraction of particles at the nuclear surface, treatment of the Pauli blocking, description of nuclear surface, collision mode and hadronisation time are discussed. The problem of the stopping time at which the cascade model gives place to the evaporation model is examined in detail. The introduction of a pre-equilibrium step, intermediate between the cascade and evaporation steps is investigated and shown to bring no significant improvement of the predictions. The production of composites and of intermediate mass fragments is shortly studied, with the help of the standard model, of the three step model mentioned above and of a cascade plus percolation model. (C) 1997 Elsevier Science B.V.
\end{abstract}

PACS: $25.40 . \mathrm{Sc} ; 25.40 . \mathrm{h} ; 28.20 .-\mathrm{v}$

Keywords: Nucleon-induced spallation reactions; Intranuclear cascade; Evaporation; Neutron yield

\section{Introduction}

The intranuclear cascade (INC) model has been proven very useful for the description of the gross features of nuclear dynamics when the latter is dominated by baryon-baryon

\footnotetext{
' On leave of absence from University of Liège, Physics Department, B5, B-4000 Sart-Tilman Liège 1, Belgium.
} 
collisions. This is for instance the case in heavy ion collisions in the $\mathrm{GeV}$ range $[1,2]$. In recent years, more sophisticated simulation methods, like BUU, QMD, BNV, ... (see Ref. [3], for instance) have been used to account for the features of the mean field dynamics, neglected in the INC model, and, by the same token, for nuclear matter equation of state effects. The latter are becoming more and more important at low energy. In the field of heavy ion physics, these new models have progressively replaced the INC model, although many aspects of the physics are dominated by collisions. As a consequence, the features of the mean field dynamics are difficult to isolate [4]. Due to its simplicity (and the high speed of the numerical applications), the INC model remains nevertheless very useful for the study of reactions where the dynamical evolution of the mean field plays a minor role. This is very likely the case in the early stage of proton (and light ion) induced reactions in the $\mathrm{GeV}$ range. In these collisions the nuclear density is only slightly and temporarily modified, as shown in Ref. [5]. Furthermore, a relatively small number of nucleons are emitted (in the cascade stage, see below) and therefore the target mean field is expected to be slightly modified only. A constant static potential approximation is thus presumably justified, at least for the calculation of most of the observables. The same situation holds in antiproton-nucleus interactions, as indicated in Refs. [6,7].

These reactions are generally well described by a two step model, the first step being provided by the INC model itself, and the second step by the evaporation model. The division of the whole collision process in two seemingly very different steps may appear as a rather ad hoc procedure. In fact, a careful analysis of the time evolution of the proton-nucleus system and ${ }^{3} \mathrm{He}$-nucleus system [8] has shown, within an INC calculation, that the system reaches, after some time, a fairly well equilibrated state. The INC model thus justifies, to some extent, the two step picture and provides with an intrinsic determination of the time separating the two steps.

The recent advent of proposals for developing accelerator driven nuclear reactors $[9,10]$ and the subsequent need for accurate nuclear reaction models, to be used for the study of particle transport in large and complex systems, have revived the interest in the cascade+evaporation model. The good global agreement reached by this model (see below) seems to be, at least in some circumstances, not sufficient for this kind of studies. A definite need for improving the INC part is rising, although an improvement of the evaporation part is also welcomed, and perhaps more desirable. Of course, an empirical ad hoc improvement of the model is perfectly possible, just by including a few free parameters to he fitted on benchmark measurements (this is a current procedure in technological applications). Alternatively, one may attempt to improve the model, by progressively introducing features of the so far neglected physics, on the basis of the present understanding of the latter and of the capacity to describe them in a simple and satisfying manner. This is the philosophy adopted in this paper. We can divide the possible improvements into three categories:

(i) Improvements linked with refinement of some features of the model. A typical example is the introduction of in-medium nucleon-nucleon cross-sections.

(ii) Improvements linked with the enlargement of the model, still keeping quasi- 
classical tools. We have here in mind, for instance, the introduction of preequilibrium models.

(iii) Improvements beyond the quasi-classical picture due to the quantum properties of the strongly interacting fermion systems.

The last category is of course the most difficult to deal with. In this paper, we will concentrate on several improvements of the first category and say a few words about those of the second category. The paper is organized as follows. In Section 2, we will describe the Liège INC model, as it was developed until about a year ago [11,12]. In Section 3, we will successively examine a set of possible improvements and critically discuss their effects. Section 4 is devoted to a discussion of a possible extension of the model beyond the two step picture. In Section 5, we briefly consider the INC model from another viewpoint, treating the mean field somehow more crudely, but allowing a simple procedure to generate fragments at the end of the interaction process. Finally, Section 6 contains a discussion of the results and our conclusion.

\section{The standard INC model}

\subsection{Description of the standard Liège INC model}

The Liège INC model for nucleon-induced interactions was first presented in Ref. [5] and and has slightly evolved, by minor improvements, to a version described in Refs. [11,12] and in more detail in Ref. [8]. For the sake of clarity, we recall here the most important features of the model:

- All particles are moving along straight line trajectories until two of them reach their minimum relative distance of approach $d_{\min }$. If $d_{\min } \leqslant \sqrt{\sigma_{\mathrm{tot}} / \pi}, \sigma_{\text {tot }}$ being the total collision cross-section, the two particles are forced to scatter elastically or inelastically, in concordance with cncrgy-momentum conservation. The final state is chosen at random in agreement with the experimental differential cross-sections (see Ref. [13] for detail).

- Nucleons, $\Delta$ 's and pions are considered. Charge states are distinguished. The parametrization of utilized cross-sections is provided in Ref. [14]. However, we introduced an inproved paranetrization of the np angular distribution. For incident lab energies larger than $300 \mathrm{MeV}$, we use the following formula:

$$
\left(\frac{d \sigma}{d \Omega}\right)_{\mathrm{cm}}=\sigma_{\mathrm{el}} N\left\{e^{B_{\mathrm{np}} t}+a e^{B_{\mathrm{np}} u}+c e^{\alpha_{\mathrm{c}} u}\right\},
$$

where $\sigma_{\mathrm{el}}$ is the elastic neutron-proton cross-section, taken from the compilation of Ref. [14], and the curly bracket gives the angular distribution in terms of the Mandelstam variables $t$ and $u$. The latter quantities are related to the center of mass polar angle $\theta_{\mathrm{cm}}$ of the outgoing neutron (relative to the direction of the incident neutron) and to the center of mass momentum $p_{\mathrm{cm}}$ by

$$
t=-2 p_{\mathrm{cm}}^{2}\left(1-\cos \theta_{\mathrm{cm}}\right), \quad u=-2 p_{\mathrm{cm}}^{2}\left(1+\cos \theta_{\mathrm{cm}}\right) .
$$




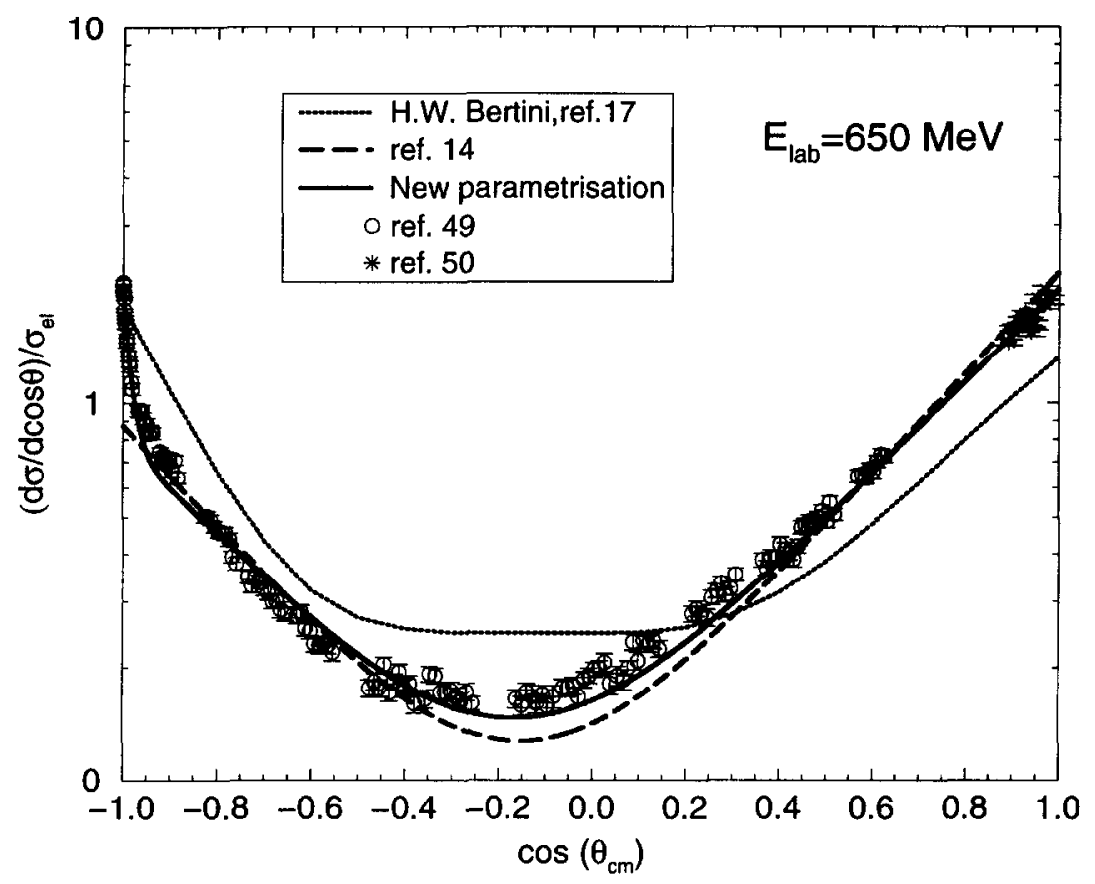

Fig. 1. Angular distribution in elastic np scattering at $650 \mathrm{MeV}$ lab energy. Comparison between experimental data [49,50] and the parametrizations of refs. [17] (dotted curve), [14] (dashed curve) and [19] (full curve, Eq. (2.1)).

The parameter $N$ is fixed by normalization, as the angular integration of Eq. (2.1) should yield $\sigma_{\mathrm{el}}$. The incident momentum dependence of the slope parameters and of the coefficients $a$ and $c$ is given by

$$
\begin{aligned}
B_{\text {np }} & =10.06-5.12 p_{\text {lab }}, & & p_{\text {lab }}<1.1 \mathrm{GeV} / c \\
& =3.68+0.76 p_{\text {lab }}, & & p_{\text {lab }}>1.1 \mathrm{GeV} / c, \\
a & =\left(\frac{0.8}{p_{\text {lab }}}\right)^{2}, & & \\
c & =6.23 \exp \left(-1.78 p_{\text {lab }}\right), & & p_{\text {lab }}<1.7 \mathrm{GeV} / c \\
& =0.3, & & p_{\text {lab }}>1.7 \mathrm{GeV} / c
\end{aligned}
$$

In these relations, the incident lab momentum $p_{\text {lab }}$ should be expressed in $\mathrm{GeV} / c$ and $B_{\mathrm{np}}$ is given in $(\mathrm{GeV} / c)^{-2}$. The third term in the curly bracket of Eq. (2.1), absent in the parametrization of Ref. [14], accounts for the rapid rise (see Fig. 1) of the cross-section at very hackward angles, due to the charged pion exchange process $[15,16]$. For incident energies less than $300 \mathrm{MeV}$, or equivalently for $p_{\text {lab }}<$ $0.8 \mathrm{GeV} / c$, the angular distribution being largely symmetric and more isotropic, we use the parametrization: 


$$
\left(\frac{d \sigma}{d \Omega}\right)_{\mathrm{cm}}=\frac{\sigma_{\mathrm{el}}}{2 \pi(2+0.05 x)}\left(1+x \cos ^{m} \theta_{\mathrm{cm}}\right),
$$

where $m=3$, if $\cos \theta_{\mathrm{cm}}>0$ and $m=4$ if $\cos \theta_{\mathrm{cm}}<0$. The numerical values of the parameter $x$ are given in Ref. [17]. For the sake of completeness, let us mention that, for the angular distribution of $\Delta$-production in nucleon-nucleon $(N N)$ collisions, we used the following form

$$
\left(\frac{d \sigma}{d \Omega}\right)_{\mathrm{cm}}=\sigma_{N N \rightarrow \Delta N} N\left\{e^{B_{\mathrm{in}} t}+e^{B_{\mathrm{in}}\left(t_{1}-t\right)}\right\},
$$

where $N$ is a normalization coefficient and $t_{1}$ is the minimum value of the $t$ variable. The value of $B_{\text {in }}$ which can be extracted from the recent measurements of Ref. [18]. It is given by [19]:

$$
\begin{aligned}
B_{\text {in }} & =5.287\left(1+\exp \frac{p_{\text {lab }}-1.3}{0.05}\right)^{-1}, & & p_{\text {lab }}<1.4 \mathrm{GeV} / c \\
& =4.65+0.706\left(p_{\text {lab }}-1.4\right), & & p_{\text {lab }}>1.4 \mathrm{GeV} / c .
\end{aligned}
$$

It only slightly differs from the value quoted in Ref. [14], which is based on fewer and fairly old data. Let us note in passing that the results of INC calculations are not sensitive to this difference. However, the crude and discontinuous angular distribution used in the Bertini code gives catastrophic results in the region of the quasi-inelastic peak (see below), as mentioned in Ref. [20] and illustrated in Ref. [19] in the $N N$ case.

- All baryons are experiencing a constant potential well of $40 \mathrm{MeV}$ depth inside the nuclear target volume.

- At $t=0$, the incident nucleon is hitting the nuclear surface and target nucleons are randomly positioned inside the target sphere (of radius $R=1.12 A^{1 / 3}$ ). Their momentum is chosen at random inside a Fermi sphere. The Fermi momentum $p_{\mathrm{F}}$ is equal to $270 \mathrm{MeV} / \mathrm{c}$.

- Collisions and 4 -decays are forbidden when the presumed nucleon final states are already occupied. In fact, this is done at random according to Pauli blocking factors. In the case of collisions with two nucleons in the final state for instance, one calculates the product

$$
P_{12}=\left(1-f_{1}\right)\left(1-f_{2}\right),
$$

where $f_{i}$ is the phase space occupation probability in the vicinity of the $i$ th nucleon. It is evaluated by counting identical particles inside a reference volume in phase space around the point $\left(r_{i}, p_{i}\right)$. The reference volume is the direct product of a sphere in $r$-space, of $2 \mathrm{fm}$ radius, and of a sphere in $p$-space, with a radius of $200 \mathrm{MeV} / c$. The collision (or decay) is blocked when $P_{12}$ is larger than a random number chosen between 0 and 1 .

- When a baryon hits the inside wall of the potential, it is reflected if its energy (kinetic+potential) is lower than the threshold. If not, it is reflected on the surface or leaves the target volume in accordance to a transmission probability $P_{\mathrm{tr}}$ given by 


$$
P_{\mathrm{tr}}=\frac{4 \sqrt{T\left(T-V_{0}\right)}}{2 T-V_{0}+2 \sqrt{T\left(T-V_{0}\right)}} e^{-2 G},
$$

where $T$ is the kinetic energy of the particle, $V_{0}$ the potential depth and $G$ the usual Gamow factor

$$
G=\frac{Z_{\mathrm{T}} z e^{2}}{\hbar c} \sqrt{\frac{2 M}{T-V_{0}}}\left(\arccos x-x \sqrt{1-x^{2}}\right),
$$

with

$$
x=\frac{T-V_{0}}{B}, \quad B=\frac{Z_{\mathrm{T}} z e^{2}}{R} .
$$

In these equations, $z$ is the charge of the incident particle, $Z_{\mathrm{T}}$ is the (actual) charge of the targct and $M$ is the mass of the particle. The total energy of the particle is conserved when it leaves the target. Thereafter, it is supposed to do not interact anymore.

- The INC is run until $t=t_{\text {fin }}$, to be discussed below. At that time the remaining $\Delta$ 's are forced to decay. The remnant or residue is defined by the nucleons inside the target volume.

- Observables are calculated by ensemble averages.

\subsection{Conservation laws}

The latter are already discussed in Ref. [5]. We recall them briefly for making the paper self-contained. Of course, baryon number and charge conservation are respected. In particular, if we denote by $A$ the target mass, $N_{\mathrm{ej}}$ the number of expelled baryons (including the incident nucleon when necessary) and $A_{\text {rem }}$ the mass of the remaining system, we have

$$
A+1=N_{\mathrm{ej}}+A_{\text {rem }} \text {. }
$$

Let us discuss now the energy conservation law. In the INC model, the energy comes from the kinetic energy of the particles, from their potential energy inside the well, from the mass of the $\Delta$ 's (with respect to the nucleon mass) and from the mass of the pions. Let $T_{0}$ and $T_{i}^{(0)}, i=1, \ldots, A$ be the kinetic energy of the incident nucleon and of target nucleons at time $t=0$, respectively. One may write

$$
T_{0}+\sum_{i=1}^{A}\left(T_{i}^{(0)}-V_{0}\right)=\sum_{j=1}^{N_{\mathrm{ej}}} \bar{T}_{j}+\sum_{l=1}^{N_{\pi}} \epsilon_{l}+\sum_{k \in A_{\mathrm{rem}}}\left(\bar{T}_{k}-V_{0}\right) .
$$

In this equation, the bar above $T$ indicates that the mass difference (with the nucleon mass) should he included in the case of a $\Delta$-particle, $N_{\pi}$ is the number of pions and $\epsilon_{l}$ is the total energy of the lth pion. Let us stress that Eq. (2.14) holds at any time. The remnant energy (last term in the r.h.s.) can be split into ground state and excitation energy. We define the former as 


$$
E_{0}=\sum_{i=1}^{A} T_{i}^{(0)}\left(\frac{A_{\mathrm{rem}}}{A}\right)^{5 / 3}-\sum_{k \in A_{\mathrm{rem}}} V_{0}
$$

Considering the second term in the 1.h.s. of Eq. (2.14) as the ground state energy of the target $E_{0}^{A}$, the difference between the two quantities can be regarded as an approximation of the actual separation energy. Indeed, for $N_{\mathrm{ej}} \ll A$, as it is often the case, one has

$$
\begin{aligned}
S=E_{0}-E_{0}^{A} & \approx \sum_{i=1}^{A} T_{i}^{(0)}\left[\left(\frac{A_{\mathrm{rem}}}{A}\right)^{5 / 3}-1\right]+\left(A-A_{\mathrm{rem}}\right) V_{0} \\
& \approx-\frac{5}{3} \sum_{i=1}^{A} T_{i}^{(0)} \frac{N_{\mathrm{ej}}-1}{A}+\left(N_{\mathrm{ej}}-1\right) V_{0} \\
& \approx\left(N_{\mathrm{ej}}-1\right)\left(V_{0}-\frac{5}{3} \sum_{i=1}^{A} \frac{T_{i}^{(0)}}{A}\right),
\end{aligned}
$$

where, in the second step, Eq. (2.13) has been used. On the average, this yields

$$
S=E_{0}-E_{0}^{A} \approx\left(N_{\mathrm{ej}}-1\right)\left(V_{0}-T_{\mathrm{F}}\right),
$$

where $T_{\mathrm{F}}$ is the kinetic energy at the Fermi surface. This is, roughly speaking, the threshold energy needed to remove $N_{\mathrm{ej}}-1$ nucleons from the target. In fact, the Fermi motion being generated at random, the corresponding value of the kinetic energy is fluctuating event by event. To avoid spurious effects due to these fluctuations, we define $E_{0}$ (see Eq. (2.16)) by the actual value of the Fermi energy and not by its average nominal value. The excitation energy can be defined correspondingly by ${ }^{2}$

$$
E^{*}=\sum_{k \in A_{\mathrm{rem}}}\left(\bar{T}_{k}-V_{0}\right)-E_{0}
$$

and Eq. (2.14) can take the form

$$
T_{0}=\sum_{j=1}^{N_{\mathrm{ej}}} \overline{T_{j}}+\sum_{l=1}^{N_{\pi}} \epsilon_{l}+E^{*}+S .
$$

Inside the INC model, momentum conservation is not fulfilled, because of the reflection of the particles on the static potential wall. Adopting the latter picture is equivalent to assume an infinitely heavy target. This expectedly introduces an error of the order of $A^{-1}$ in the calculated momentum of an ejectile. The r.m.s. error in the total momentum of the ejected particles is then of the order of $\sqrt{N_{\mathrm{ej}}} A^{-1}$. The final remnant momentum can be estimated with about the same accuracy through the relation

\footnotetext{
${ }^{2}$ This equation and the following one are slightly difierent from those of Ref. [5], where the energy of the incident particle, or better, of the leading particle is singled out. In the energy range considered in this paper, the leading particle goes out of the target almost surely and the final value of $E^{*}$ remains the same as in the present formulation.
} 


$$
\boldsymbol{p}_{0}=\sum_{j=1}^{N_{\mathrm{ej}}} \boldsymbol{p}_{j}+\sum_{l=1}^{N_{\pi}} \boldsymbol{p}_{l}+\boldsymbol{p}_{\mathrm{rem}} .
$$

The final remnant recoil energy is therefore given (non-relativistically) by $T_{\text {rem }}=$ $p_{\text {rem }}^{2} /\left(2 A_{\text {rem }} M\right)$. The calculated excitation energy can be corrected for this contribution by using

$$
E^{*}=\sum_{k \in A_{\text {rem }}}\left(\bar{T}_{k}-V_{0}\right)-E_{0}-T_{\text {rem }} .
$$

In the results below, this is not introduced. As a matter of fact, $T_{\text {rem }}$ is generally very small, reaching a few $\mathrm{MeV}$ at the most. Note, however, that $p_{\text {rem }}$ may be a sizable fraction of $p_{0}$, as large as one half in typical cases.

\subsection{The stopping time}

The characteristic time evolution of the various quantitics appcaring in Eq. (2.19) is discussed in Ref. [5]. Therein it is shown that the target excitation energy raises rapidly when the incident particle penetrates the target, reaches a maximum after a few $\mathrm{fm} / c$ and decreases first rather quickly (by emission of fast particles). After some time, the excitation energy decreases further, on a much slower rate, akin to the evaporation process. The idea is thus to stop the cascade at this time and switch on an evaporation calculation. This procedure is not only introduced for saving computation time (avoiding running the cascade for very long times). It seems to be necessary, as the evaporation pattern is known to depend sensitively on the level density, which is presumably different from the single particle model value, to which the INC model implicitly corresponds.

In Ref. [8], it is shown that the change of slope in $E^{*}(t)$ is correlated with similar changes in the time variation of many other quantities. This observation enables us to determine a stopping time $t_{\text {fin }}$ at which the cascade is terminated. The changes of slopes being not instantaneous, the quantity $t_{\text {fin }}$ is not sharply defined: uncertainties of $2-5 \mathrm{fm} / c$ or less are present. As a simple rule, we determine $t_{\text {fin }}$ as the value corresponding to the crossing of the two characteristic straight lines, fitting the time variation of $E^{*}(t)$ in the two regimes, in a semi-logarithmic plot [8]. It is smoothly depending upon target mass,incident energy and impact parameter. We do not produce the exact value here. Just to give an idea, $t_{\text {fin }} \approx 25 \mathrm{fm} / c$ for $\mathrm{p}(1 \mathrm{GeV})+\mathrm{Pb}$ central collisions. It is roughly proportional to $A^{1 / 3}$ and only slightly dependent upon the energy in the $0.4-1.2 \mathrm{GeV}$ range.

\subsection{Illustrative results}

We give in Figs. 2 and 3, the comparison between the predictions of the INC model described above and the experimental data for two systems and three energies. In these figures, only the INC contribution is shown, the evaporation giving a modification at small neutron energy, practically not visible on the scale of the figures (see the discussion below). The overall agreement is remarkable, in view of the simplicity of the 


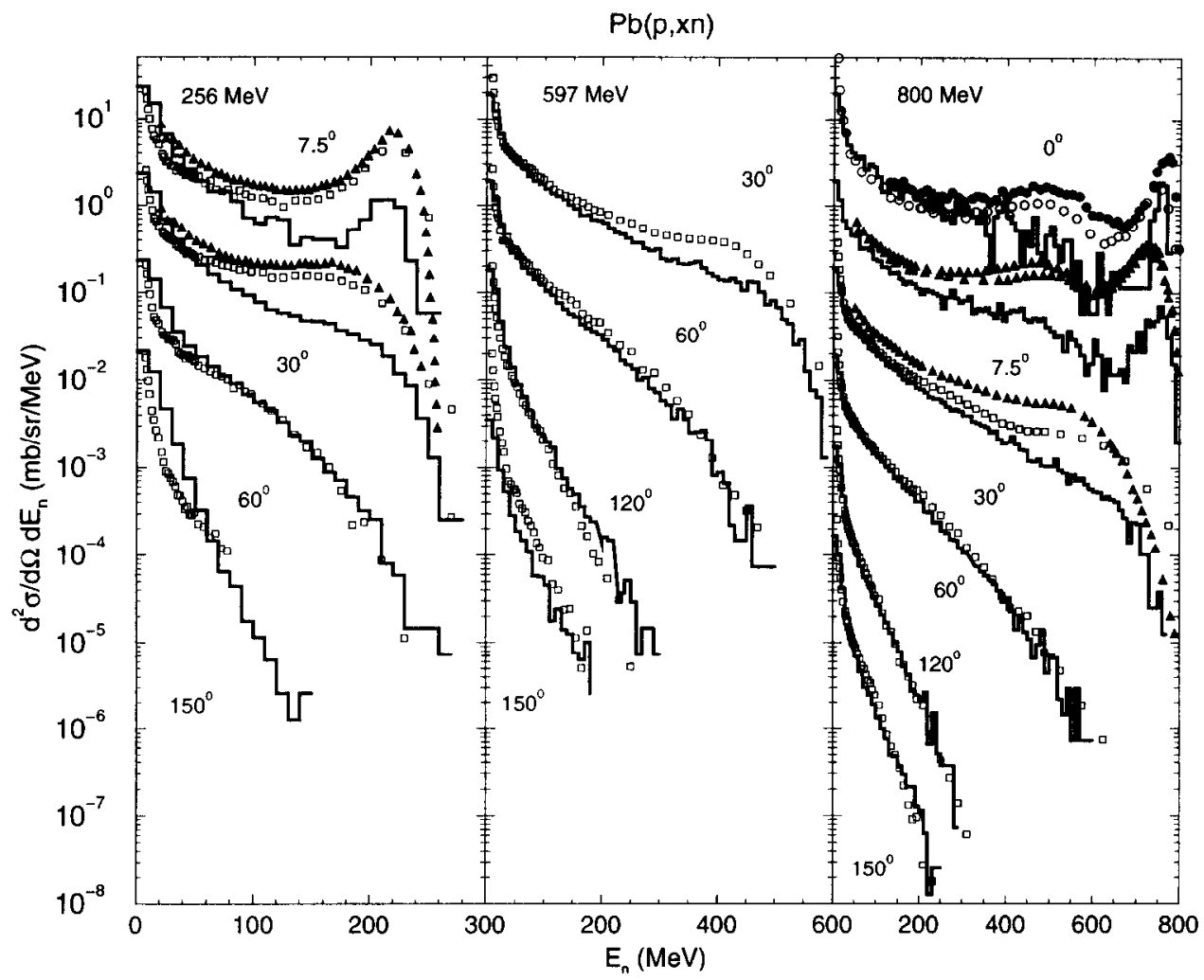

Fig. 2. Neutron double differential cross-sections for $\mathrm{p}+\mathrm{Pb}$ reactions at 256,597 , and $800 \mathrm{MeV}$. Comparison between the results of the Liège INC model (histograms) and experiment (symbols). Data are taken from Refs. [18,51-55]. In order to avoid superposition of curves, only the histogram and the data for the smallest angle are given in absolute value. The other ones have been multiplied by $10^{-1}, 10^{-2}, \ldots$ for the other angles, in increasing order. Experimental error bars are not shown, but are of the order of, or smaller than the size of the symbols. The theoretical results correspond to the simulation of $\sim 10^{5}$ events.

model. Nevertheless, systematic discrepancies appear (we purposely presented Figs. 2 and 3 in a linear-log form in order to enlighten these discrepancies, which could easily escape from the attention of the reader in $\log -\log$ plots, often used in the literature to present similar results). The neutron double differential cross-section is underestimated for the $\mathrm{Pb}$ target at small angles and for the $\mathrm{Fe}$ target at $30^{\circ}$, both for large neutron energies, corresponding roughly to the quasi-elastic and the quasi-inelastic processes. In the former, the incident proton hits a neutron elastically, which leaves the nucleus without any further interaction. In the latter process, the incident proton is excited to a $\Delta$-resonance, whereas the neutron is still emitted without further interaction. This is illustrated by Fig. 4, which shows the calculated neutron energy spectrum at some angle for the $p(800 \mathrm{MeV})+\mathrm{Pb}$ case along with the contribution from the ejected neutrons having suffered one collision only. The lack of yield of high energy neutrons at relatively small angles seems to come from a too strong re-interaction in the cascade model. Fig. 5 shows the comparison with the recent measurements of Martinez et al. 


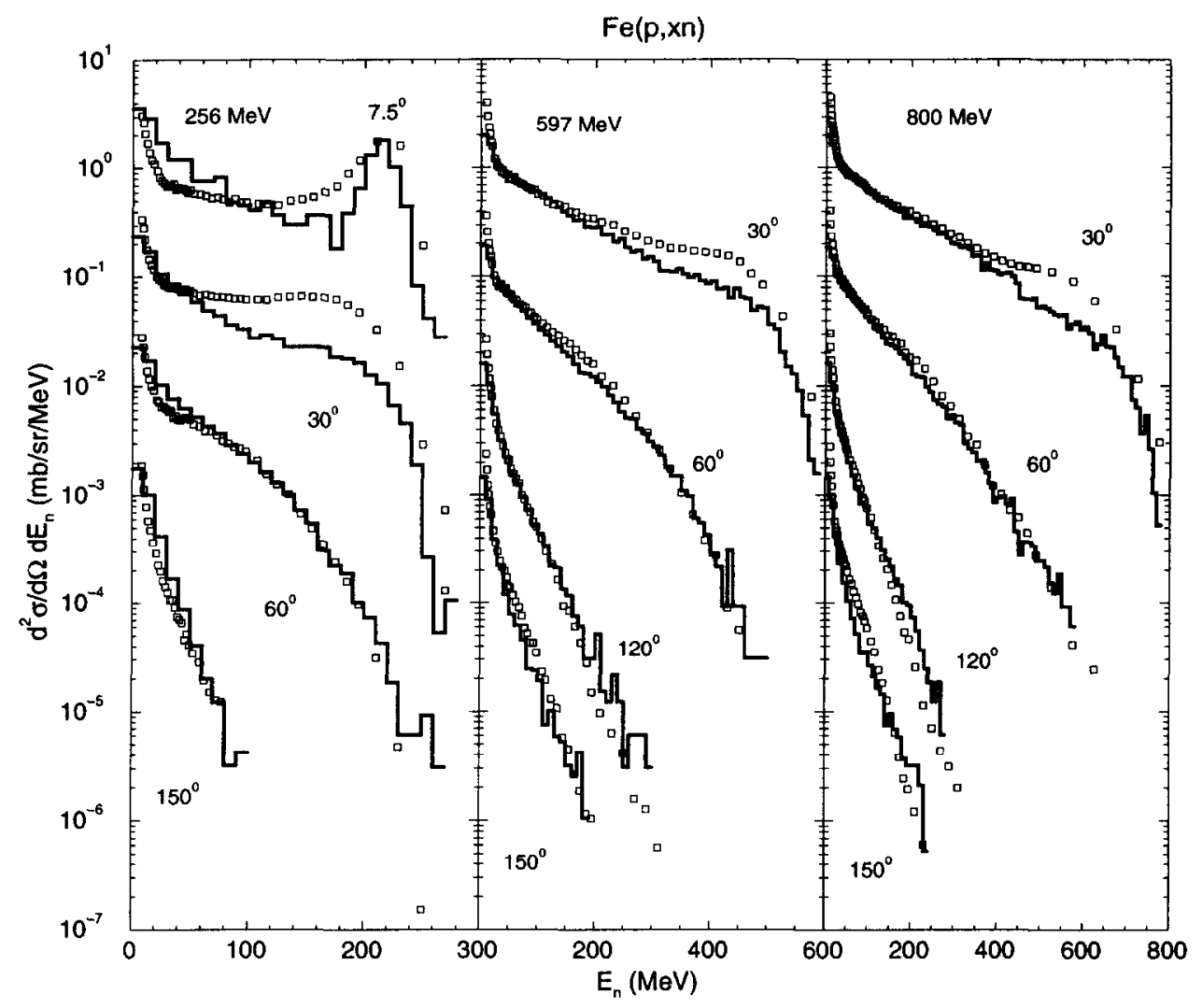

Fig. 3. Same as Fig. 2 for a Fe target. Data (symbols) are taken from Refs. [52,56,57].

[18] at $0^{\circ}$ and $1200 \mathrm{MeV}$ incident energy. Once again, the agreement is satisfactory, but a small discrepancy clearly shows up. The width of the quasi-elastic and the quasiinelastic peaks are too small, by a factor 2 or so. This possibly points out quantum effects (single-particle strength, smearing of the Fermi surface, etc) which can be at work in this kinematical region. It might appear paradoxical that the single-scattering component, which is expectedly suited to be well described by quasi-classical approaches as the INC model, is in fact not as well reproduced as the bulk of the spectra below, say $400 \mathrm{MeV}$, involving much softer collisions, for which, a priori, quantum effects are more important. Cancellations of quantum effects after many collisions could provide a plausible explanation of this paradox. We will come back to this point in the conclusions.

Typical low energy spectra calculated with the INC+evaporation model are shown in Fig. 6, where the splitting between the INC and evaporation components is displayed. The evaporation part is calculated with the numerical code of Dresner et al. [21], taking the level parameter density as $a=A / 10$ throughout this paper. Several comments are of order. First the overall agreement is quite good. Each component is overwhelmingly dominant in a well defined domain, which can easily be identified. Finally, there seems to be no place for a third component. We will elaborate on this point later on. 


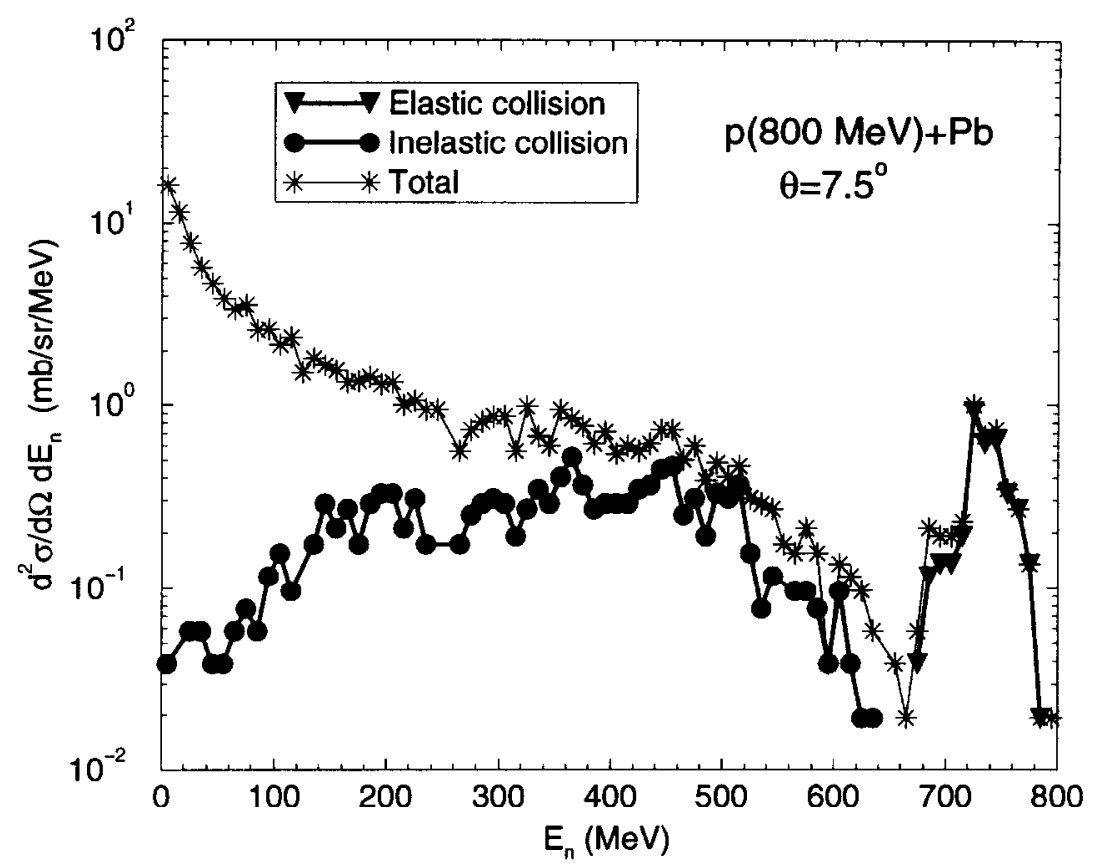

Fig. 4. Comparison between the calculated total neutron double differential cross-section and the contribution from the neutrons having made a single collision. The figure refers to neutrons emitted at $7.5^{\circ}$ in the $\mathrm{p}(800 \mathrm{MeV})+\mathrm{Pb}$ system. Triangles and heavy dots refer to elastic and inelastic single scattering respectively. See text for detail.

A typical residue mass spectrum, calculated with the INC+evaporation model, is exhibited in Fig. 7 and compared with the measurements of Ref. [22]. Although the interpretation of the results is not always obvious (calculations deal with all residues after the evaporation stage, summed over families of isobars, whereas data refer to $\gamma$ activities, which sum over the isobars of $\beta$-decay chains following the evaporation), the predictions are in reasonably good agreement with the data. In the particular case of Fig. 7, the mass spectrum can be divided into three parts: a huge peak near the target mass value (the so-called spallation peak), a smaller peak due to symmetric fission (centered around $A \sim 80$ ), and a low mass component (not shown in the figure). The first peak comes from neutron evaporation by the excited residue left over by the cascade. The more excited this residue is, the broader the spallation peak is. Fig. 7 shows that the calculation using the Bertini model yields a too large spallation peak. This indicates that the remnant is too much excited in this model, since the same evaporation code is used with the two calculations. The difference of the calculated fission peak has presumably the same origin. 


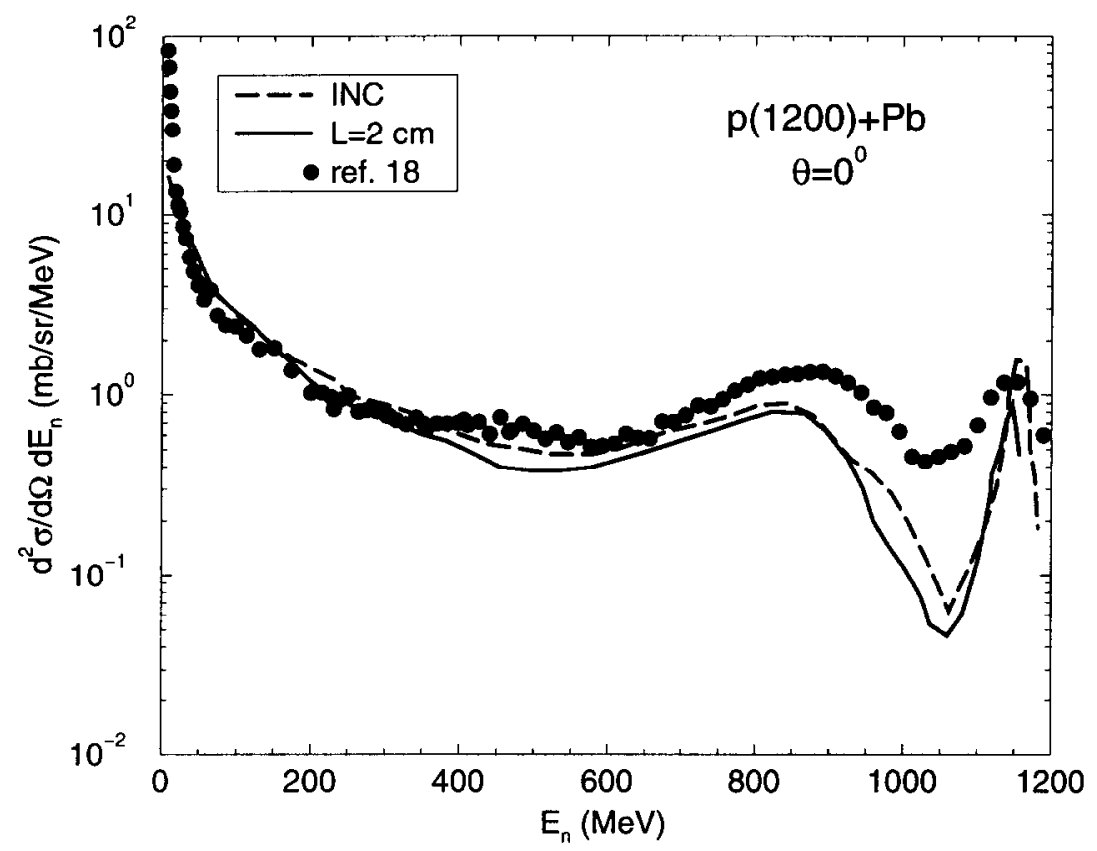

Fig. 5. Neutron double differential cross-section at $0^{\circ}$ for $\mathrm{p}+\mathrm{Pb}$ reactions at $1200 \mathrm{MeV}$. Comparison between the results of the Liège INC model and experiment. Data (dots) are taken from Ref. [18]. Experimental error bars are not shown, but are of the order of, or smaller than the size of the symbols. Theoretical results are given by the curves: the dashed line corresponds to an infinitely thin target, the full line is obtained after correction for a target thickness of $2 \mathrm{~cm}$ as in the experiment. They are obtained from running $\sim 10^{5}$ events.

\section{Study of possible improvements of the INC model}

\subsection{Introduction}

We successively discuss below some potential improvements of the INC model, either introducing new physical aspects not contained in our standard version, or refining the treatment of some particular points of the latter. We mainly limit ourselves to showing and discussing the effects of each of the modifications on the neutron spectra and sometimes on the residue mass spectrum (for the former, we only investigate the cascade component). We will postpone the general discussion to the Section 6. Let us however make a comment on the new parametrization of the np elastic cross-section. For convenience, we consider it as part of the definition of the standard version of the Liège INC model, although it was not included in previous published calculations. The main effect of using the previous parametrization [14] (see Fig. 1) is a sizable reduction of the quasi-elastic peak, but at very small angles only. 


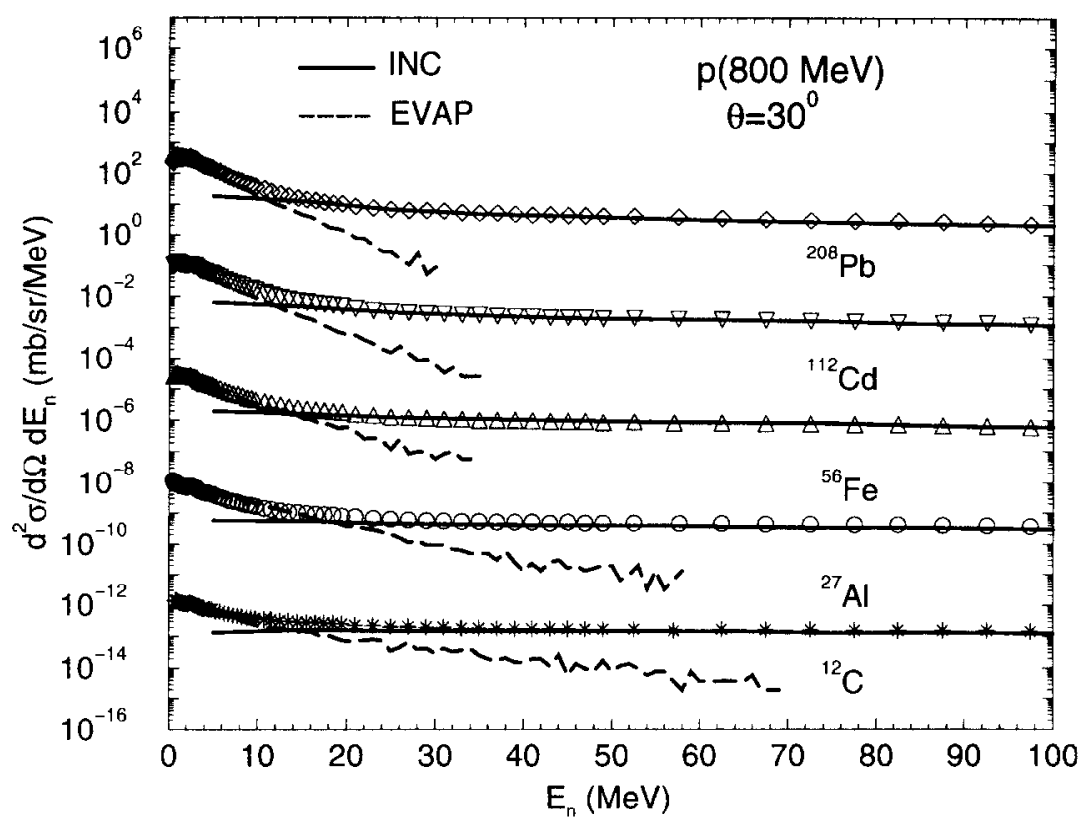

Fig. 6. Neutron double differential cross-sections at $30^{\circ}$ for $p$-induced reactions at $800 \mathrm{MeV}$ on various targets. Comparison between the results of the INC+evaporation model and experiment. Data (symbols) are taken from Ref. [52]. In order to avoid superposition, only the curves and the data relative to the heaviest target are given in absolute value. The other ones have been multiplied by $10^{-3}, 10^{-6}, \ldots$ for the other targets in decreasing order. Experimental error bars are not shown, but are of the order of, or smaller than the size of the symbols. Theoretical results, split into INC contribution (full curves) and evaporation contribution (dashed curves), correspond to the simulation of $\sim 10^{5}$ events using the standard Liège cascade and the evaporation code of Ref. [21].

\subsection{In-medium cross-sections}

Baryon-baryon collision cross-sections inside nuclear medium differ from free space cross-sections. It is generally believed [23] that the modification arises from a softening of the short range part of the nucleon-nucleon interaction potential, due to the presence of the underlying Fermi sea, as embodied by the Brueckner $g$-matrix [24]. This is substantiated by the recent progress in the derivation of the nuclear Boltzmann-like transport equation $[25,26]$. In Fig. 8 , we show the in-medium elastic cross-sections, calculated in Refs. [27-29]. In all calculations the method amounts to replace the free $T$-matrix by the Brueckner $g$-matrix, although there are minor differences in the definition of the flux and of the phase space density. However, the results of Refs. [27,28] are based on relativistic calculations, whereas the results of Ref. [29] are obtained in a non-relativistic framework. Results are quite different, especially for the $\mathrm{np}$ case.

Fig. 9 shows the output of an INC calculation using the in-medium cross-sections of Refs. [27,28]. We chose the latter, as they correspond to a larger reduction of the cross-sections, in order to show what is presumably an upper limit of medium effects. For energy beyond the range of Fig. 8 , we assume that the free cross-sections are 


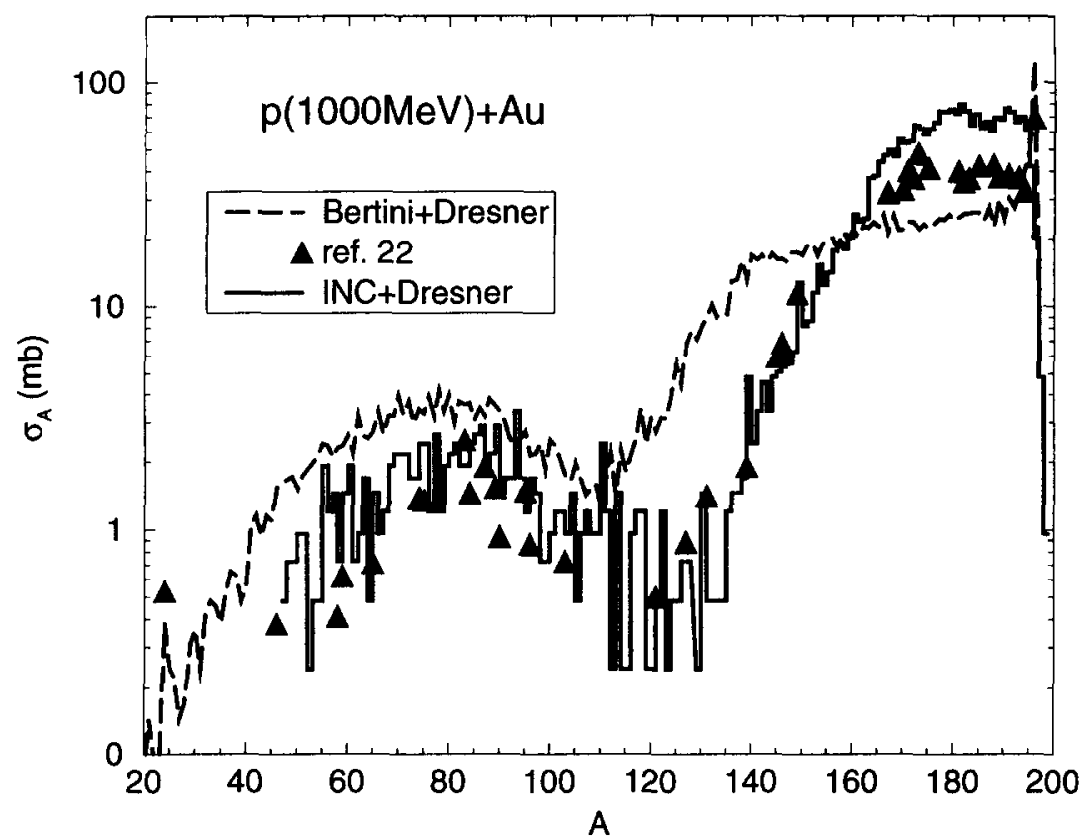

Fig. 7. Residue mass spectrum after $p(1 \mathrm{GeV})+{ }^{197} \mathrm{Au}$ reactions. Data (symbols) are taken from Ref. [22]. Theoretical results (histograms) are obtained in a INC+evaporation model, summing over $\sim 10^{5}$ events; full lines correspond to the standard Liege cascade and dashed lines, to the Bertini model. In both cases, the evaporation model of Ref. [21] is used.

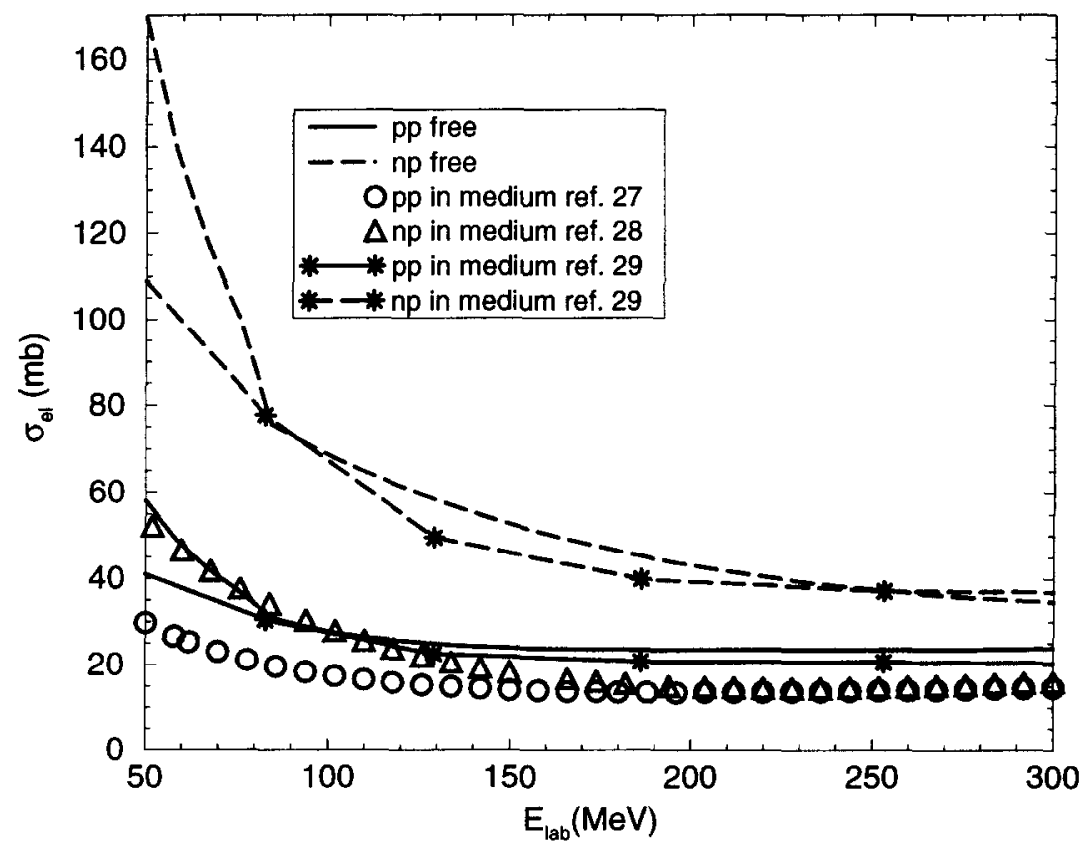

Fig. 8. Values of the free and in-medium elastic cross-sections. The latter are taken from Refs. [27-29]. 


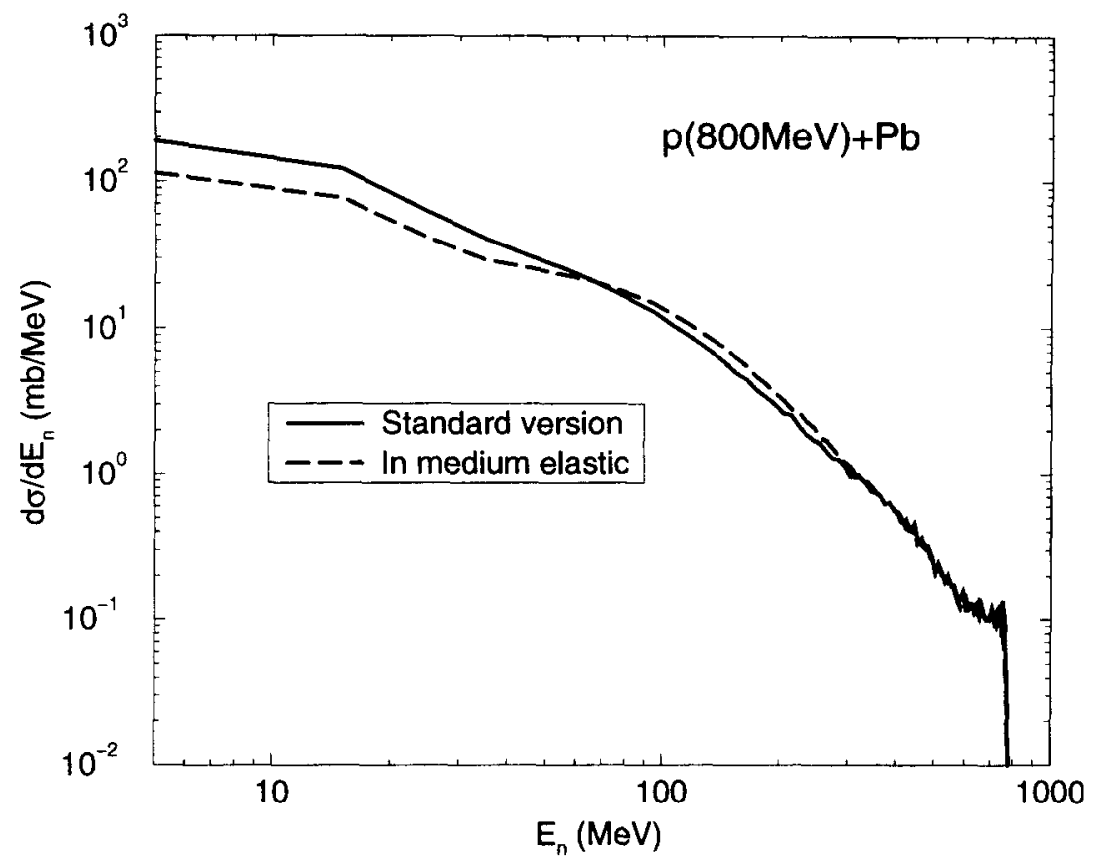

Fig. 9. Angle integrated neutron spectrum in $\mathrm{p}(800 \mathrm{MeV})+\mathrm{Pb}$ reactions. Comparison between the results of the standard Liège INC model and the results obtained using the in-medium elastic cross-section of Refs. $[27,28]$.

recovered smoothly around $E_{\mathrm{lab}}=400 \mathrm{MeV}$. The most remarkable feature of Fig. 9 is the depletion of the neutron yield at small neutron energy. There is practically no effect at high neutron energy, as expected: the in-medium reduction of the cross-sections disappears for energetic collisions. Of course, the global effect is expected to increase at lower incident energy [30].

We also investigated a little bit the effect of the in-medium modification of the inelastic cross-sections. The latter have been calculated by ter Haar and Malfliet [31], still within Brueckner theory: at normal nuclear matter density, the reduction is about a factor $1 / 2$ or so, except close to the inelastic threshold, where there is no reduction. We checked that at $800 \mathrm{MeV}$, the main effect on the neutron spectra is a reduction of the quasi-inelastic peak, by about $40 \%$.

\subsection{Refraction at the nuclear surface}

In the standard version, nucleons are either reflected or transmitted along a straight line, when they hit the surface. We made a calculation, allowing the refraction of the nucleons at the surface. Calling $p_{\mathrm{r}}$ and $p_{\mathrm{t}}$ the radial and tangential momenta of a particle of kinetic energy $T$ hitting the surface, and $p_{\mathrm{r}}^{\prime}$ and $p_{\mathrm{t}}^{\prime}$ their values after transmission, the usual laws of refraction demand

$$
p_{\mathrm{r}}^{\prime 2}=p_{\mathrm{r}}^{2}+V_{0}^{2}-2(T+M) V_{0}, \quad p_{\mathrm{t}}^{\prime}=p_{\mathrm{t}},
$$




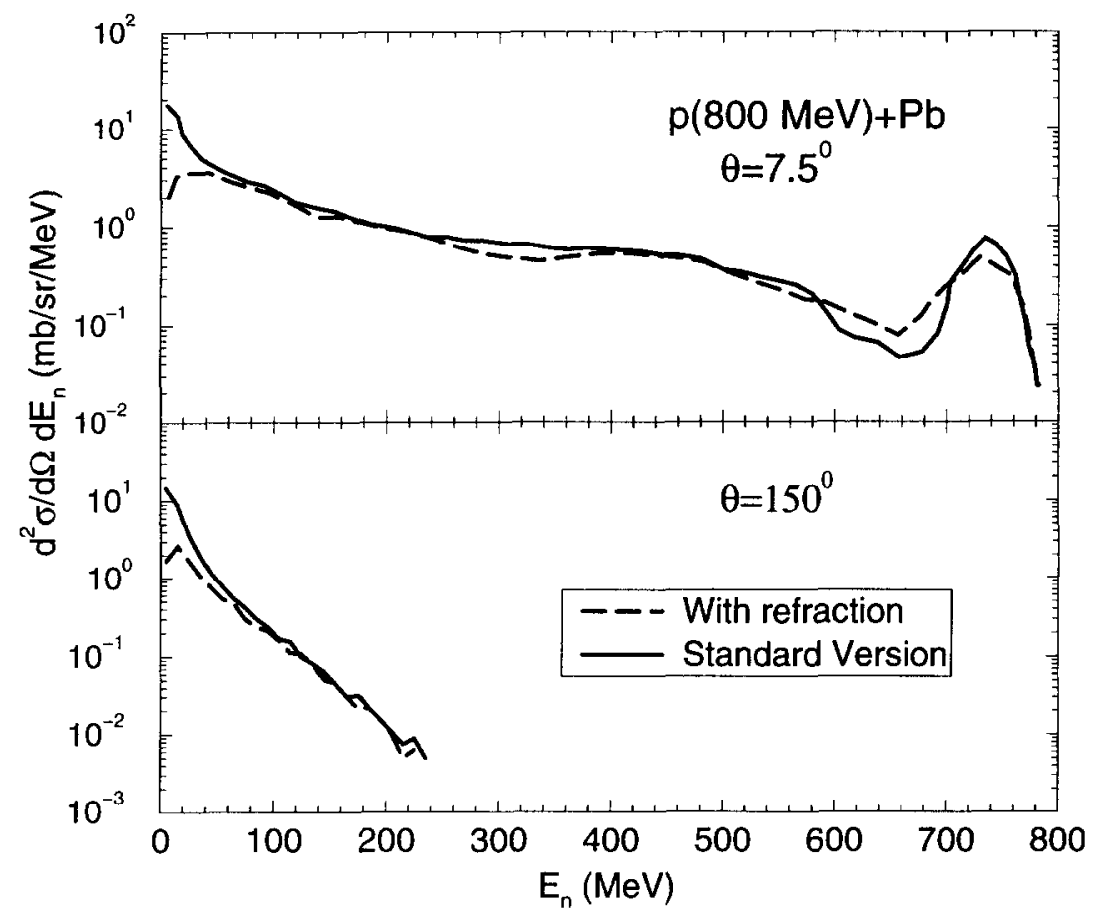

Fig. 10. Neutron double differential cross-sections at $7.5^{\circ}$ and $150^{\circ}$, in $\mathrm{p}(800 \mathrm{MeV})+\mathrm{Pb}$ reactions. Comparison between the results of the standard Liège INC model and the results obtained after introducing refraction of the particles on the nuclear surface. See text for detail.

where $V_{0}$ is the potential depth. As a consequence, total reflection occurs when the incidence angle is larger than the Brewster angle. The latter is then given by

$$
\theta_{\mathrm{B}}=\arctan \sqrt{\frac{\left(T-V_{0}\right)\left(2 M+T-V_{0}\right)}{V_{0}\left(2 M+2 T-V_{0}\right)}} .
$$

This angle is larger and larger when the energy is increasing. The main result of this modification is a strong reduction of the yield of low energy neutrons, as shown by Fig. 10. This is due to the vanishingly small Brewster angle for low energy outgoing neutrons $\left(T \sim V_{0}\right)$. Let us notice that, in this calculation, the transmission probability has been put equal to unity, when total reflection does not occur. Using a transmission coefficient as in the standard cascade would give even more dramatic effects. As it can be surmised from Fig. 10, the energy integrated neutron yield is more focused to forward angles than in the standard case.

\subsection{Stopping time}

This is not a priori a source of improvement, since the value of the stopping time can be determined in a consistent way, as we explained in Section 2. However, we want to make a few comments on the changes brought by possible modifications of the 


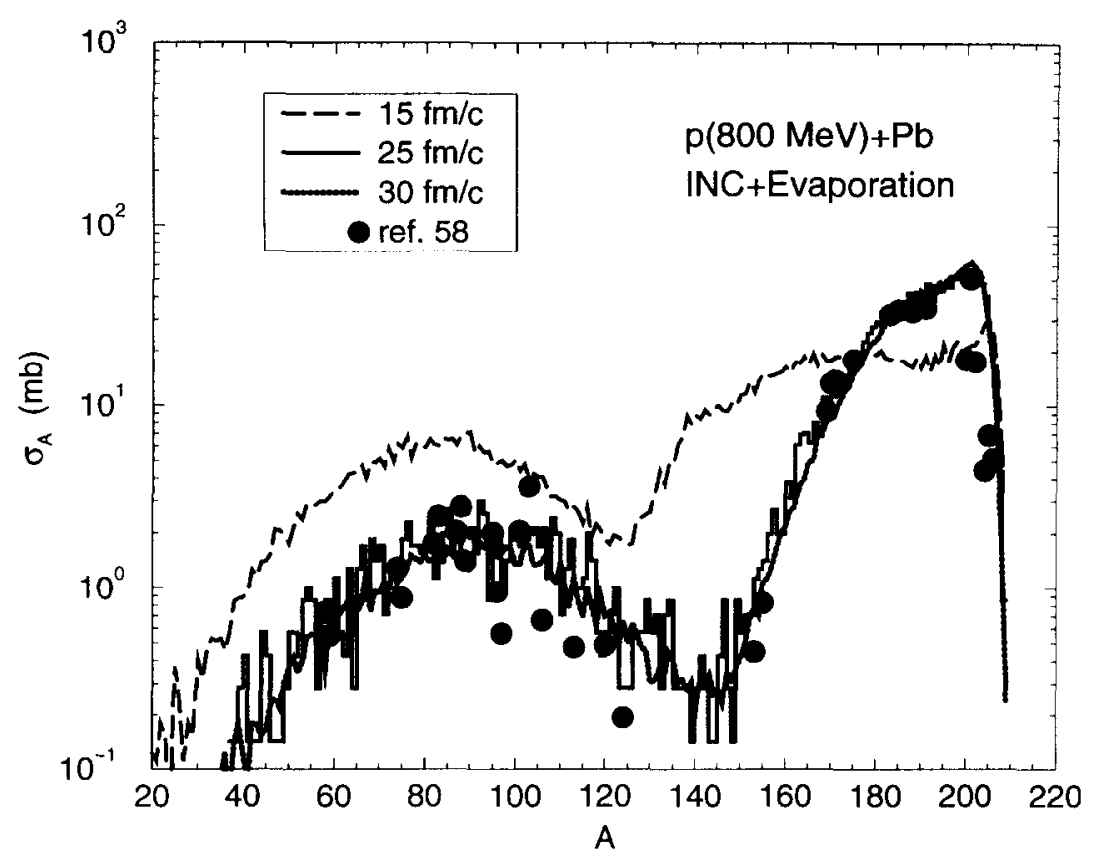

Fig. 11. Residue mass spectrum after $\mathrm{p}(800 \mathrm{MeV})+\mathrm{Pb}$ reactions. Data (symbols) are taken from Ref. [58]. Theoretical results (histograms) are obtained in the standard Liège INC+evaporation model, for the standard value and for two extreme values of the stopping time $t_{\mathrm{fin}}$ of the cascade.

stopping procedure. The stopping time being of the order of $25 \mathrm{fm} / c$ (for heavy targets at $800 \mathrm{MeV}$ ) [8], reasonable changes of this value can be a few $\mathrm{fm} / c$ at the very most. When $t_{\text {fin }}$ is changed by $5 \mathrm{fm} / c$, the average number of emitted cascade particles $(\simeq 7)$ changes by $\sim 1.5$ units and the average excitation energy $(\simeq 130 \mathrm{MeV})$ changes by $\sim 40 \mathrm{MeV}$. Therefore, if the cascade is stopped earlier, by $\sim 5 \mathrm{fm} / c$, the number of cascade nucleons is decreased by $\sim 1.5$ units and the excitation energy is increased by $\sim 40 \mathrm{MeV}$, which will be converted in $\sim 2$ extra neutrons in the evaporation stage (the converse is true if the cascade is stopped later). The total number of emitted particles is only slightly different from what it is for the standard value of $t_{\text {fin }}$. The neutron spectra are hardly changed either, as the particles which are emitted at the end of the cascade are not very energetic, whereas the neutrons which are emitted at the beginning of the evaporation are more energetic than those at the end of this process. Both kinds of neutrons have roughly $\sim 10 \mathrm{MeV}$ kinetic energy (this is rather evident from Fig. 6).

The stability against modifications of $t_{\text {fin }}$ is less valid for the residue mass spectrum. In fact, the latter results from the subsequent evolution, driven by the evaporation, of the mass-energy distribution generated by the cascade. This evolution is rather complex and somehow sensitive to changes of the initial (post-cascade) distribution [32]. This is illustrated by Fig. 11. Of course, here variations of $t_{\text {fin }}$ are extreme, but the larger sensitivity (compared to the one of the neutron spectra) is obvious. It is interesting to note that increasing $t_{\text {fin }}$ beyond the standard value makes a lesser change to the mass 
spectrum than decreasing it, reflecting the fact that the excitation energy is varying more slowly for $t$ larger than the standard value of $t_{\text {fin }}$.

It might be of interest to comment on the comparison between our INC code and the so-called Bertini code [17]. The main distinction (at least in relation to the present discussion) is the fact that the latter model has no time structure: only the trajectories of the active particles are generated on the basis of a mean free path with an exponential law and terminated when the particle is ejected or when its kinetic energy inside the target is smaller than the Fermi energy plus some amount $\epsilon$. This parameter can be chosen at will, but it is usually taken as $8 \mathrm{MeV}$. We remind that active particles are those that have been struck (and promoted from the inactive Fermi sea continuum) by the incident particle or by another active particle. We investigated the results of the two codes at the end of the cascade. We found, within our model, an average excitation energy smaller by $\sim 40 \%$ and a larger number of emitted particles, by $\sim 4$ units, in the typical $p(800 \mathrm{MeV})+\mathrm{Pb}$. Choosing $t_{\text {fin }} \approx 15 \mathrm{fm} / c$ yields roughly the same output as in the Bertini code. Trials to determine a value of $\epsilon$ for which the Bertini code yields about the same results as our code (for the standard value of $t_{\text {fin }}$ ) remained unsuccessful. It seems that the correspondence $t_{\text {fin }} \leftrightarrow \epsilon$ does not hold for all values. Presumably, this comes from the fluctuations which are apparently much larger in our code than in the other one. We have no solid explanation for this, although it is tempting to attribute it to the explicit description of the Fermi motion in our model. In any case, we have good confidence in the appropriateness of our choice for $t_{\mathrm{fin}}$. Besides the fact mentioned in Section 2 that the change of regimes is signalled by simultaneous changes in the time variation of many quantities [8] and that this choice avoids any extrinsic arbitrariness, we produce another argument in Fig. 12. The latter shows the time evolution of the anisotropy of the momentum distribution of the nucleons inside the target, evaluated in two ways:

$$
\begin{aligned}
& A_{1}=\frac{\left(\sum_{i} p_{z i}\right)^{2}-\frac{1}{2}\left(\left(\sum_{i} p_{x i}\right)^{2}+\left(\sum_{i} p_{y i}\right)^{2}\right)}{\left(\sum_{i} p_{z i}\right)^{2}+\frac{1}{2}\left(\left(\sum_{i} p_{x i}\right)^{2}+\left(\sum_{i} p_{y i}\right)^{2}\right)} . \\
& A_{2}=\frac{\sum_{i} p_{z i}^{2}-\frac{1}{2} \sum_{i}\left(p_{x i}^{2}+p_{y i}^{2}\right)}{\sum_{i}\left(p_{z i}^{2}+p_{x i}^{2}+p_{y i}^{2}\right)} .
\end{aligned}
$$

These quantities are averaged over events. Of course, event by event, their departure from zero may be much larger, but it is reassuring to see that both quantities are practically vanishing for $t \gtrsim t_{\text {fin }}$, indicating that the remnant is fairly randomized at $t \approx t_{\text {fin }}$.

In an attempt to understand the differences between the two codes, we realized a "Bertini-equivalent" version of our own code, forbidding nucleon-nucleon collisions "inside" the Fermi sea in two ways: (i) We forbid any target nucleon to collide with another target nucleon before hitting the potential wall or before colliding with the incident nucleon, if this occurs earlier. (ii) We forbid any target nucleon to collide, except with the incident nucleon or with an already active nucleon (here, a nucleon becomes active as soon as it has collided with the incident nucleon or with another active 


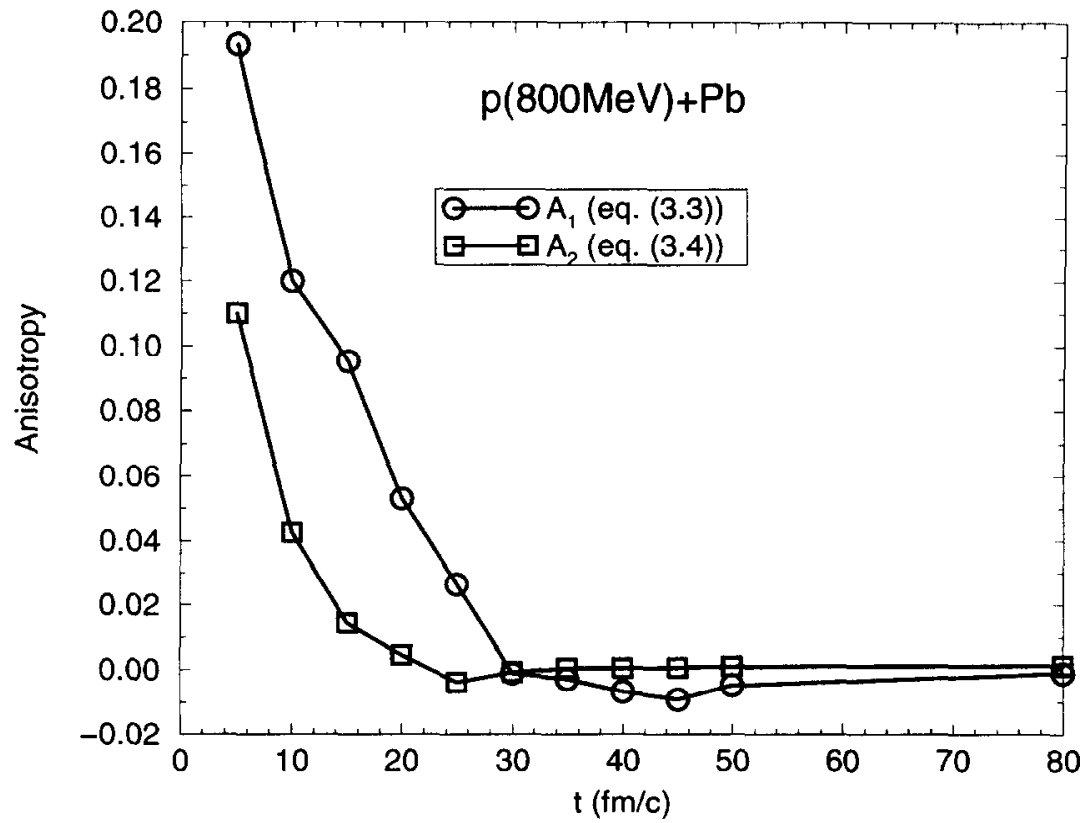

Fig. 12. Time evolution of the anisotropy of the momentum distribution for the nucleons remaining in the target in the standard Liège INC model, as calculated using Eqs. (3.3) and (3.4).

particle). We remind that, in our code, collisions inside the Fermi sea, even "cold", may occur, just because the occupation probability $n(p)$ is a discrete function of $p$ and because of fluctuations inherent to the stochastic description of the Fermi motion and of the Pauli blocking. Modification (i) does not bring any significant change, whereas the second one reduces sizably the number of emitted soft particles, as shown in Fig. 13. This observation is however hard to interpret. It is sure that in our standard INC code, the imperfect realization of Fermi motion and Pauli blocking unduly promotes particles above the Fermi sea. On the other hand, the sharp Fermi surface and the strict Pauli blocking adopted in the Bertini code disregard the dynamical distortion and depletion of the Fermi sea, as the collision process unfolds. Let us finally note that, in an actual nucleus, even in the heaviest ones, the momentum distribution does not present a sharp boundary. We do not have any clear cut criterion to determine in which case the error is the smallest, except for the quality of the comparison with experiment.

\subsection{Pauli blocking}

We investigate several modifications of the Pauli blocking scheme explained in Section 2. But, before entering the discussion, it is important to assess, as far as possible, the importance of the Pauli blocking and the quality with which it is implemented in the standard cascade. First, we can state that the number of blocked collisions is of the order of $40 \%$ and the number of blocked $\Delta$-decays is of the order of $5 \%$. If the Pauli blocking is suppressed, the number of ejected particles is increased by $\sim 35 \%$ and the 


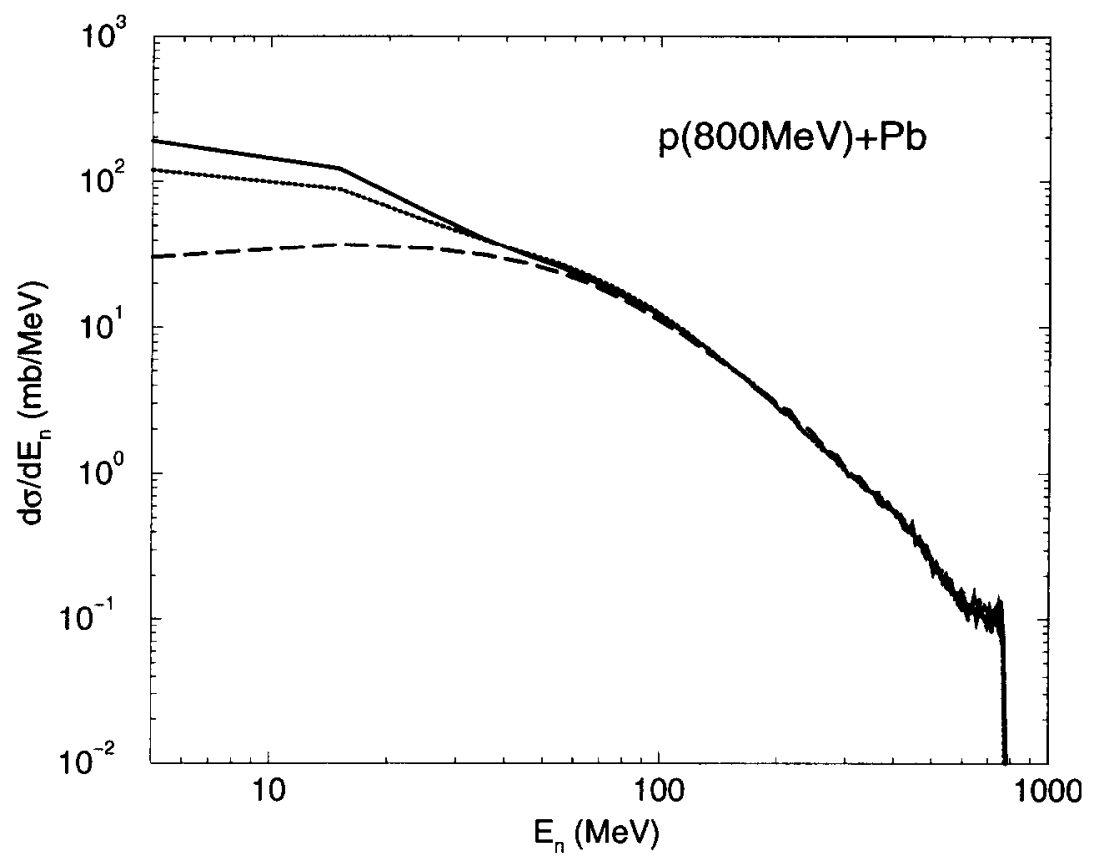

Fig. 13. Angle integrated neutron spectrum in $\mathrm{p}(800 \mathrm{MeV})+\mathrm{Pb}$ reactions. Comparison between the results of the standard Liège INC model (full line) and the results obtained after further reducing collisions between particles inside the Fermi sea. The dotted line and the dashed line refer to modifications (i) and (ii) mentioned in the text, respectively.

shape of the spectrum is enhanced at small neutron energy (see Fig. 14), in agreement with the discussion in the previous subsection. The quasi-inelastic and the quasi-elastic contributions are increased as well, by almost a factor 2 for the latter. Surprisingly, the spectrum is noticeably depleted in the $60-120 \mathrm{MeV}$ range.

The quality of the Pauli blocking in the standard cascade is testified by Fig. 15, which shows the occupation number distribution in momentum space for the nucleons inside the remnant (as a function of the kinetic energy). The expected shape of a thermally excited (unconfined) Fermi sea is showing up, rather nicely. The asymmetric distortion around the Fermi level is due to the fact that particles excited above the Fermi level have, with a good chance, escaped from the system (note however that the depletion just below the Fermi level seems to be too strong). The occupation number is abnormally too large at the bottom of the Fermi sea, near $p \approx 0$. This defect is not as dramatic as it may appear at first sight since it involves a very small fraction of the particles only. Let us notice that even the best methods used up to now in transport models, as those involving a huge number of test particles per nucleon, suffer from the same defect [33]. Altogether, in view of the rather crude recipe used in our standard cascade, the handling of the Pauli blocking is quite remarkable. One has to realize that the distribution shown in Fig. 15 is far from the Boltzmann occupation number distribution corresponding to the same available energy. The latter is an exponentially decreasing function of $E$ with 


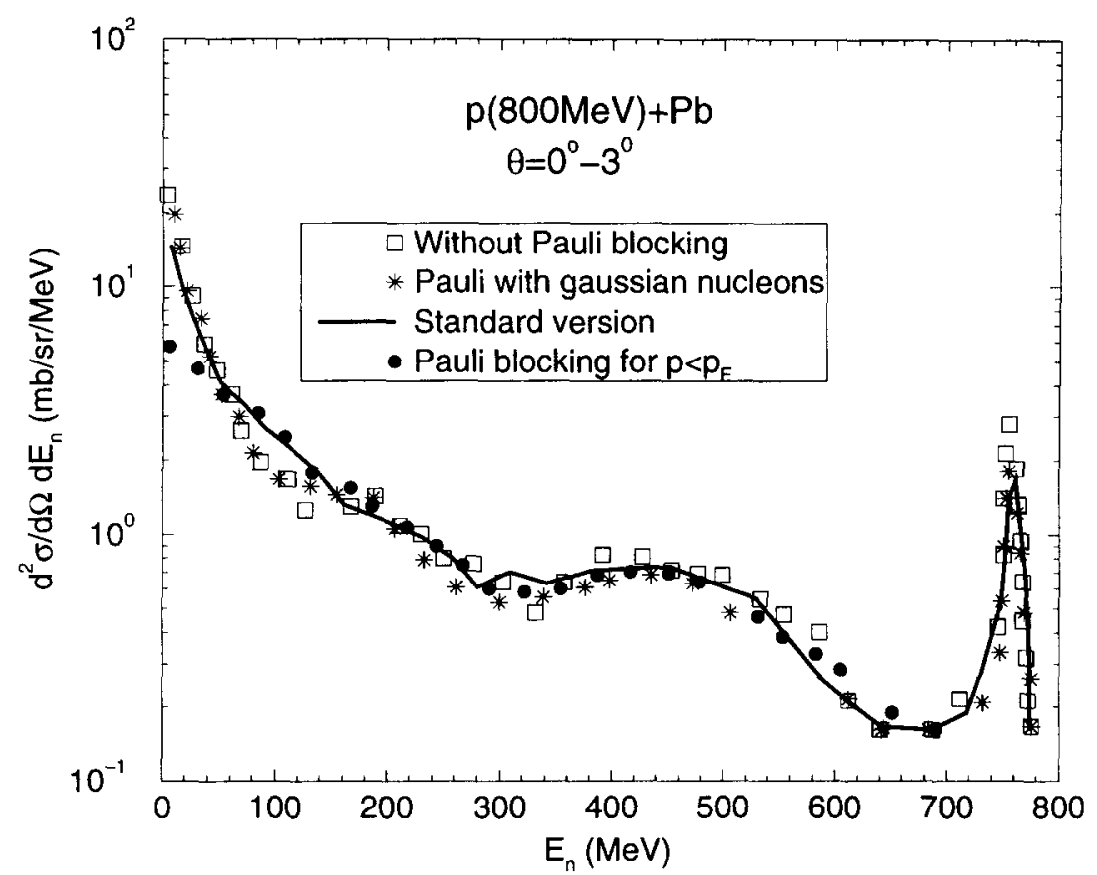

Fig. 14. Neutron double differential cross-sections summed over angles between $0^{\circ}$ and $3^{\circ}$, in $\mathrm{p}(800 \mathrm{MeV})+\mathrm{Pb}$ reactions. Comparison between the results of the standard Liège INC model, the results obtained by totally relaxing the Pauli blocking and those obtained by using two alternative methods for implementing the Pauli blocking. See text for detail.

a slope parameter equal to $\sim 16 \mathrm{MeV}$.

In order to improve the description of the Pauli blocking, we attached to the particles, when evaluating the occupation factors $f_{i}$ (see Section 2), Gaussian profile functions with width parameters equal to $2 \mathrm{fm}$ in $r$-space and $250 \mathrm{MeV} / c$ in momentum space. The results are shown in Fig. 14 for the $\mathrm{p}(800 \mathrm{MeV})+\mathrm{Pb}$ system in the $0-3^{\circ}$ range, the importance of the Pauli principle being the largest at forward angles. The differences are disappointingly rather small in the quasi-inelastic and quasi-elastic peaks. Surprisingly, the yield is increased at small energy ( $E \lesssim 20 \mathrm{MeV}$ ) and is decreased around $100 \mathrm{MeV}$. This result is rather embarrassing, as the predictions of the standard cascade arc rather good in the intermediate energy range. For the sake of comparison, we also made a calculation forbidding collisions if one of the final momenta is below $p_{\mathrm{F}}$ (as in the Bertini code). The results are very close to those with the standard choice, except that the number of soft particles is strongly reduced, a result in agreement with our previous discussion.

\subsection{The nuclear surface}

In order to generate a non-vanishing surface thickness, we used the following recipe. We generated the Fermi motion as usual, but for a particle of momentum $p$, we determine 


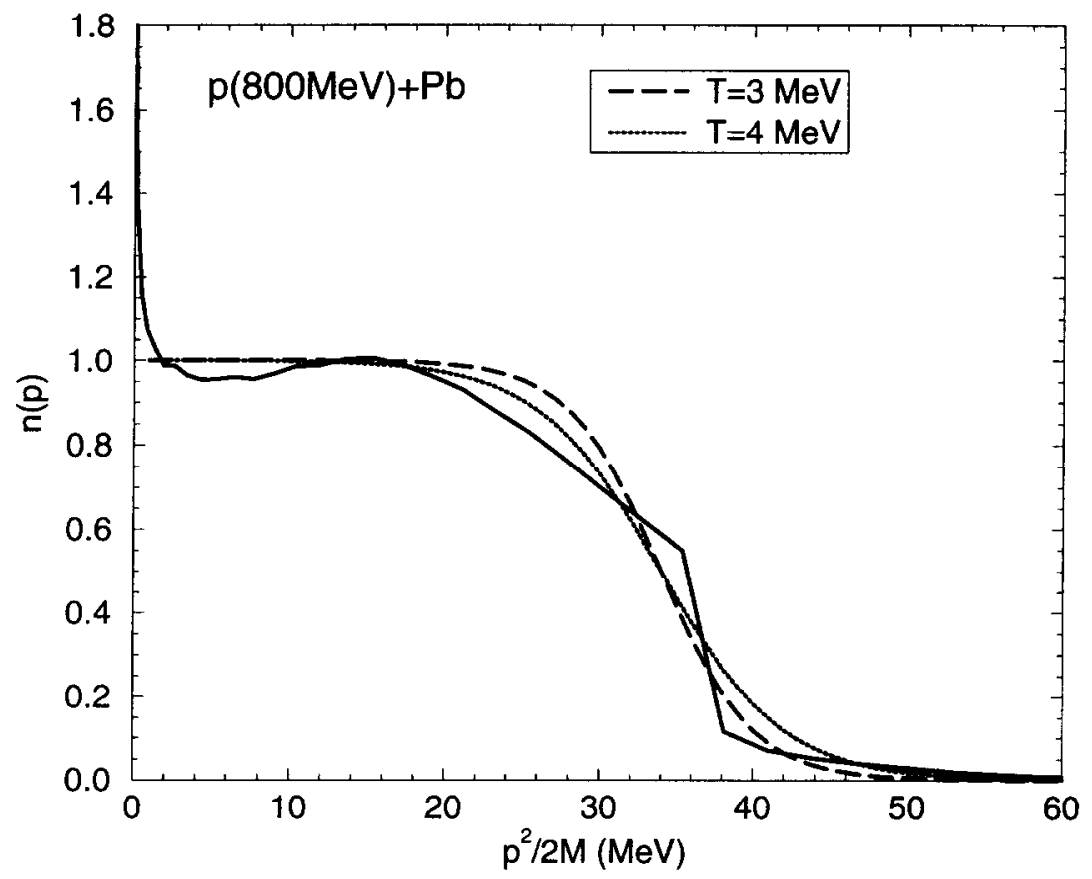

Fig. 15. Occupation number distribution for nucleons remaining inside the target volume at the end of the standard Liège INC model (full curve), compared to Fermi-Dirac distributions for two typical values of the temperature (dotted and dashed lines) and chemical potential corresponding aprroximately to the final number of particles. The abscissa represents the nucleon kinetic energy. See text for detail.

its position by using a uniform law in a sphere of radius $R_{\mathrm{s}}(p)$ related to $p$ by:

$$
\left(R_{\mathrm{s}}(p)\right)^{3}=(R-2.2 a)^{3}+\left((R+2.2 a)^{3}-(R-2.2 a)^{3}\right)\left(\frac{p}{p_{\mathrm{F}}}\right)^{3},
$$

where $a(=0.6 \mathrm{fm})$ is the diffuseness parameter. In addition, we assumed that a nucleon of momentum $p$ feels the potential well of depth $-V_{0}$ for $r<R_{\mathrm{s}}(p)$, when $p \leqslant p_{\mathrm{F}}$, and for $r<R_{\mathrm{s}}\left(p_{\mathrm{F}}\right)$, when $p>p_{\mathrm{F}}$. As a result, the quasi-elastic and quasi-inelastic peaks are enhanced by $30 \%$, the intermediate neutron energy yield is increased even more at small angles, whereas the low energy yield is decreased. This is shown in Fig. 16 and, of course, reflects the increased importance of the scattering in the low density regions. However, the total integrated neutron yield is decreased by $\sim 20 \%$.

\subsection{The collision mode}

Here we investigate the modifications of the way two body collisions are described numerically. First, it should be noticed that angular momentum is not conserved by collisions, in the standard cascade, as the momenta are changed suddenly, while the positions are not modified. We made a calculation enforcing angular momentum conservation, by moving nucleons, in their reaction plane, as shown in Fig. 17. There are two 


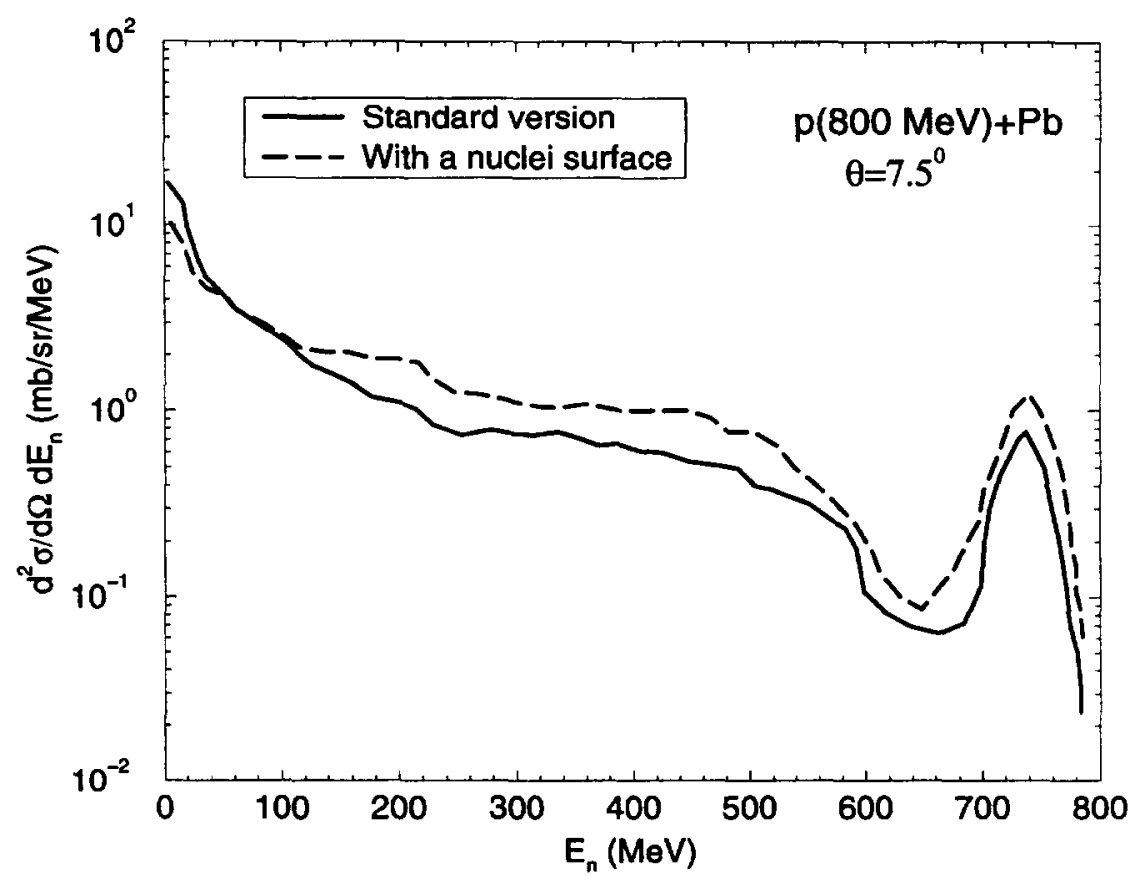

Fig. 16. Neutron double differential cross-sections at $7.5^{\circ}$ in $\mathrm{p}(800 \mathrm{MeV})+\mathrm{Pb}$ reactions. Comparison between the results of the standard Liège INC model and the results obtained by introducing a diffuse nuclear surface.
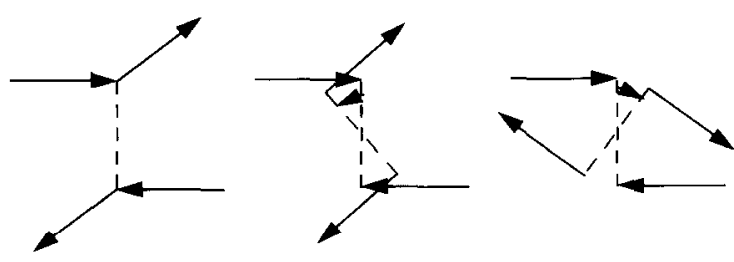

\section{Standard Repulsive Attractive}

Fig. 17. Schematic illustration of the collision style used in the standard Liege INC model (left) and the two possible, repulsive (center) and attractive (right), styles used when enforcing angular momentum conservation in binary collisions.

possible ways of doing this, either by forcing the particles to come closer to each other (the attractive style) or go away from each other (the repulsive style) in their center of mass frame. The polar angle is determined by the differential cross-section law as in the standard INC model. We recall that in the latter, the azimuthal angle with respect to the reaction plane is chosen at random. When angular momentum is conserved the latter can only be either 0 or $180^{\circ}$. We adopted here the repulsive style, because it can simulate in some way the repulsive part of the nucleon-nucleon potential [34]. The difference with the standard cascade (not shown) is amazingly small, even for the angular momentum left in the target (at least for the average value).

Alternatively we looked also at the possibility of simulating an infinitely hard core. In 


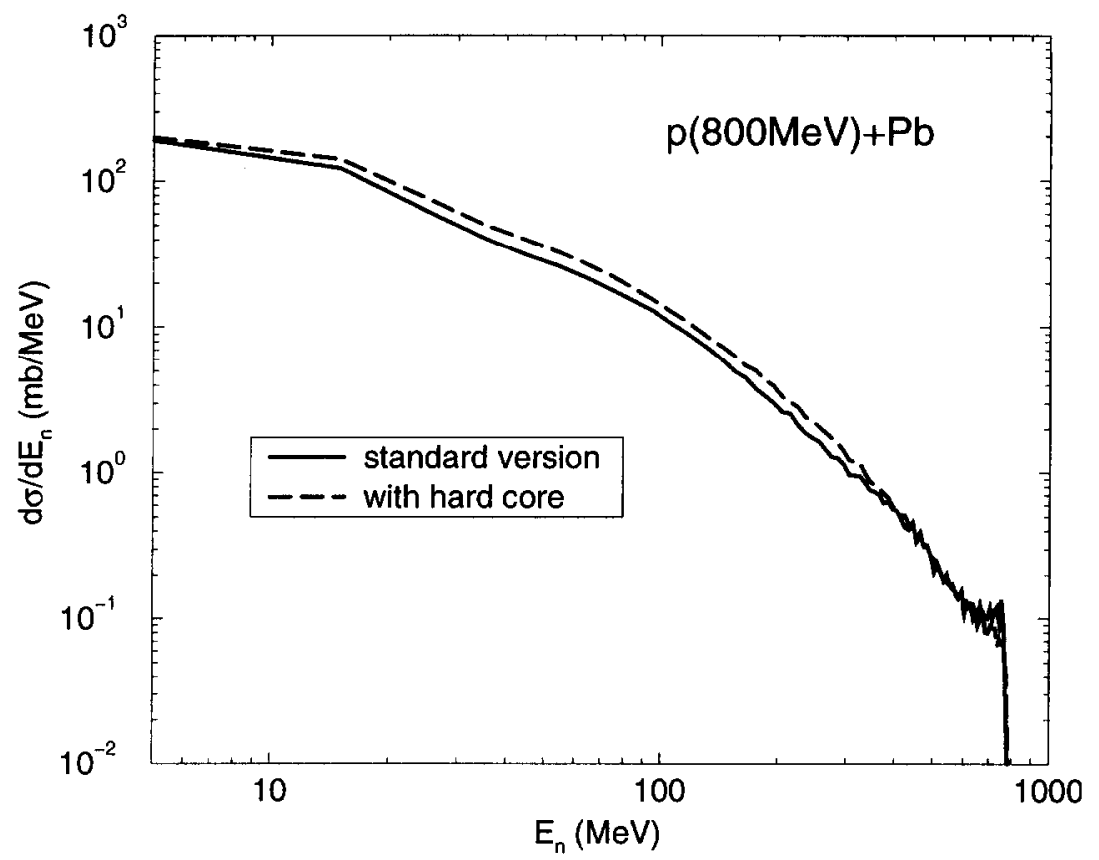

Fig. 18. Angle integrated neutron spectrum in $\mathrm{p}(800 \mathrm{MeV})+\mathrm{Pb}$ reactions. Comparison between the results of the standard Liège INC model and those obtained with a short range hard core. See text for detail.

that case, the nucleons are forced to scatter when their relative distance is equal to $r_{\mathrm{c}}$, the core radius (taken equal to $0.4 \mathrm{fm}$ ), if this happens before reaching the minimum value $d_{\text {min }}$. In that case, we impose the repulsive style, without however moving the particles. The results are shown in Fig. 18. The high energy part is slightly reduced, whereas the rest of the energy spectrum is slightly enhanced. We have no simple explanation of this result.

These two modifications are bringing insignificant changes, indicating that the particle, momentum and energy flows are largely dominated by the number of collisions and the gross features of the differential cross-sections and not so much by the detail of the particle trajectories. We finally look at another modification, which on practical grounds appears as follows. After a collision, the two outgoing particles are not allowed to interact with another particle during a (proper) time interval of size $t_{\mathrm{H}}$. Newly formed $\Delta$ 's cannot decay during this time either. This time interval $t_{\mathrm{H}}$ may be viewed as a practical trick to account for the collision time [37] or even as an effective medium effect, hindering quasi three-body collisions, i.e. two successive collisions involving the same nucleon and separated by a very short time interval. It can be viewed also as an "hadronisation time", i.e. the time after which the correlations inside hadrons have led to a well defined hadron state, as it is often considered at high energy [35,36]. At high energy, this effect is enhanced by Lorentz time dilatation, but if it is relevant, there is no reason to consider it at lower energy, except perhaps for elastic scattering, where internal correlations are probably less important. This issue is far from being settled, but 


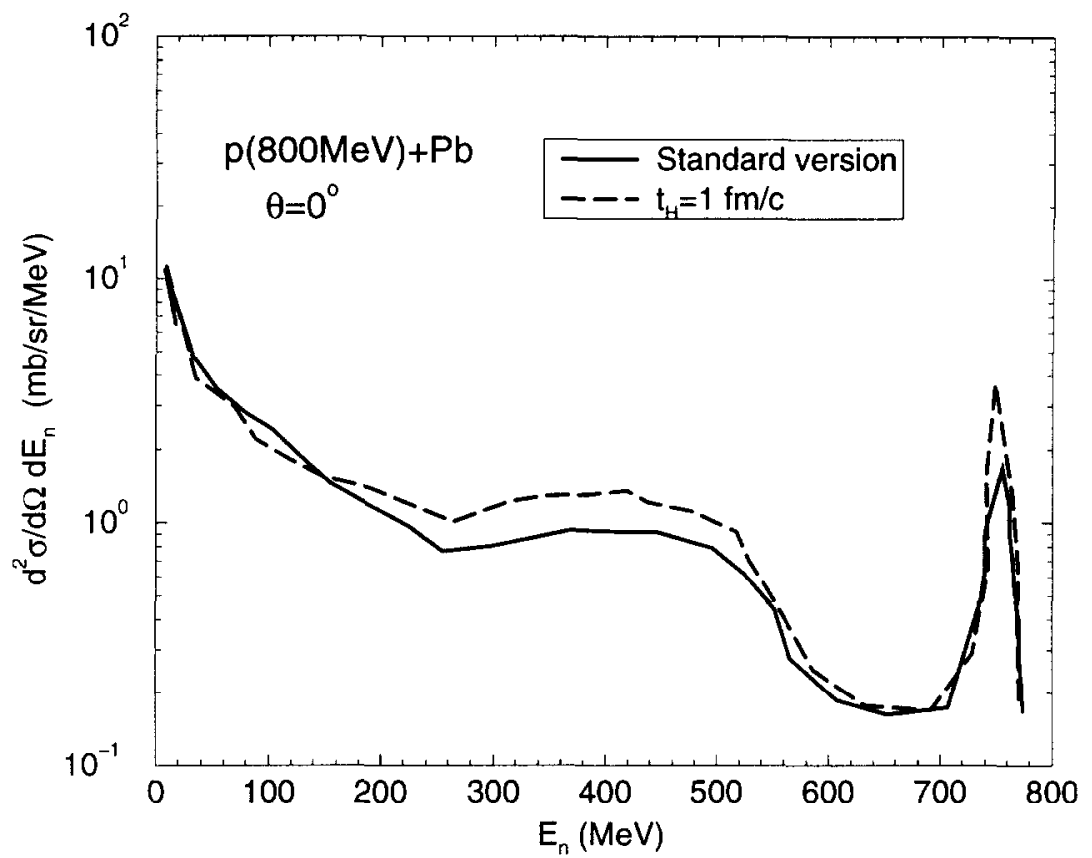

Fig. 19. Neutron double differential cross-sections summed over angles between $0^{\circ}$ and $3^{\circ}$, in $\mathrm{p}(800 \mathrm{MeV})+\mathrm{Pb}$ reactions. Comparison between the results of the standard Liège INC model and the results obtained with the introduction of a hadronisation time $t_{\mathrm{H}}=1 \mathrm{fm} / c$. See text for detail.

we want to consider it here as a possibility and evaluate its effect on proton-nucleus collisions. If this interpretation is valid, the value of $t_{\mathbf{H}}$ should not be large, otherwise it would leave some noticeable effect at low energy. On the other hand it should be of the order of the characteristic times of the internal motion of hadrons, at the most. We can thus take $1 \mathrm{fm} / c$ as an upper limit. We show in Fig. 19 the results when taking $t_{\mathrm{H}}=1 \mathrm{fm} / c$, for the neutron spectrum. The neutron yield is enhanced (by $\sim 50 \%$ ) at high energy, whereas it is slightly depleted in the $40-100 \mathrm{MeV}$ energy range. This is perhaps not surprising: the net effect is an increase of the transparency for high encrgy particles, whereas there is practically no change for slow particles, $t_{\mathrm{H}}$ being then smaller than the time separation between successive collisions.

\section{Beyond the two step model}

The sudden switching from the cascade to the evaporation schemes may appear as not physically appealing and calls for a framework where the hard processes typical of the beginning of the cascade up to the very soft processes at the end of the evaporation are treated on the same footing. This framework does not exist for the time being. As an alternative, one may consider to introduce a third stage between cascade and evaporation, that would render the evolution softer. This step could be described by one of the various versions of the so-called pre-equilibrium models (see Refs. [38,39] for an overview). 


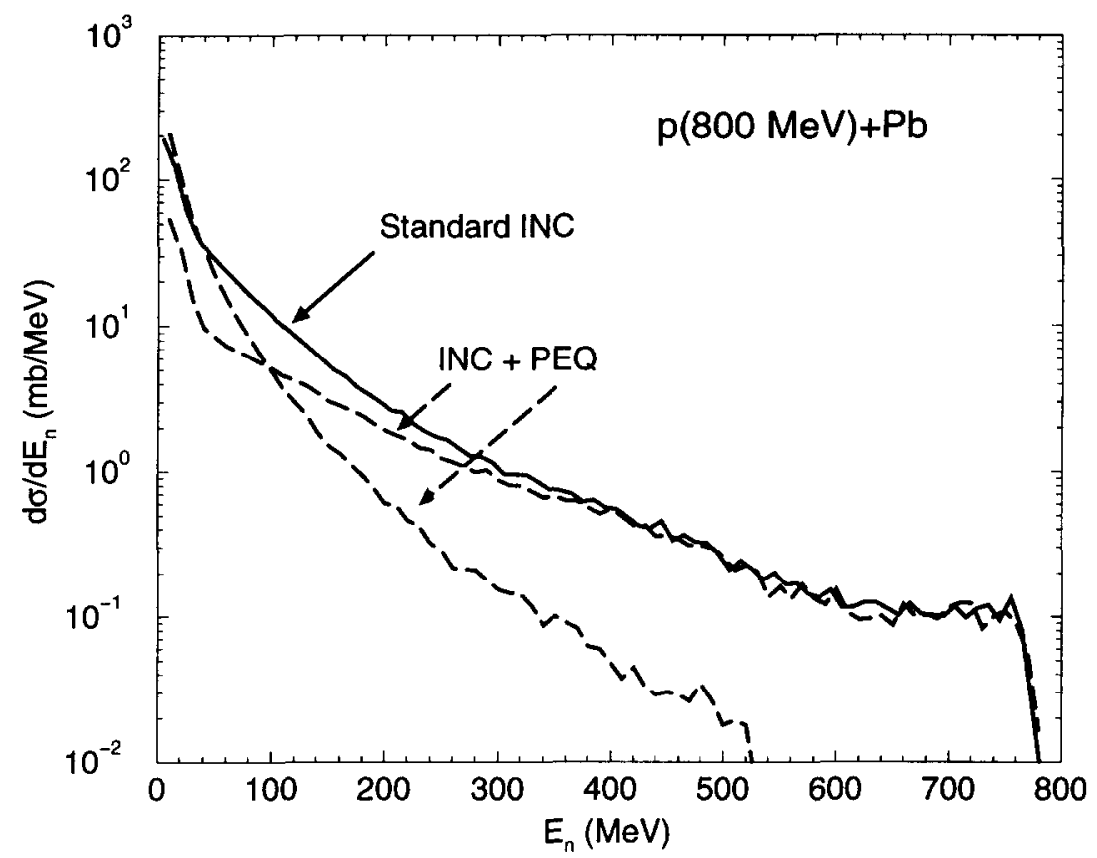

Fig. 20. Angular integrated neutron spectrum in $\mathrm{p}(800 \mathrm{MeV})+\mathrm{Pb}$ reactions. Comparison between the results of the standard Liège INC model and those obtained from an INC+pre-equilibrium model, split into the INC and the pre-equilibrium components. In this last case, the standard Liège INC model is used but stopped earlier and the pre-equilibrium model of Ref. [40] is used. See text for detail.

Without entering into the detail, these models describe the evolution of the occupation probabilities in discrete nucleon states, or more simply in exciton states. Mathematically, this evolution is governed by a set of master equations. The transition probabilities are related in one way or the other to the nucleon-nucleon collision cross-sections. In addition, escape probabilities are introduced to describe the emission of nucleons (using basically transmission factors) and, in some cases, of light composites (using essentially ad hoc parameters). The evolution is followed until thermal equilibrium is reached. Beyond this point the standard evaporation is assumed. Roughly speaking, these models carry the same physics as the INC. They differ however on two points: (i) they use a discrete single-particle spectrum rather than a continuous one; on the other hand they generally abandon the directional description of the motion of the particles (ii) they allow an easy phenomenological account of the emission of composites. Note that the introduction of the pre-equilibrium model is possible at the expense of the introduction of an undetermined time parameter separating the INC and the pre-equilibrium steps. Therefore it is not a priori evident that the introduction of this step is necessary or useful. In order to investigate this point, we performed a calculation for the $p(800 \mathrm{MeV})+\mathrm{Pb}$ system, stopping the cascade a little bit earlier (at $\sim 20 \mathrm{fm} / c$ for central collisions), when the decrease of the excitation energy departs from the first exponential fall-off, and turning to the pre-equilibrium model of Mashnik (see details in Ref. [40]). We show 
Table 1

Average particle multiplicities in INC+evaporation, INC+pre-equilibrium+evaporation and INC + percolation+evaporation models for the $\mathrm{p}(800 \mathrm{MeV})+\mathrm{Pb}$ system. The brackets indicate the average over events. The symbols $n$ and $p$ refer to free neutrons and protons, respectively, and the indices refer to their origin. The entries of the fifth and sixth rows correspond to non-evaporative neutrons. The symbol $N_{\text {free }}$ refers to nucleons. The quantity $\boldsymbol{M}_{\text {comp }}$ is the multiplicity of composite particles. For the second model, the multiplicity of composites has been split into the pre-equilibrium and evaporation components

\begin{tabular}{|c|c|c|c|}
\hline & Standard INC+evaporation & INC+ + pre-eq. + evap. & INC+ + perc. + evap \\
\hline$\left\langle n_{\mathrm{INC}}\right\rangle$ & 5.02 & 1.51 & 1.92 \\
\hline$\left\langle n_{\mathrm{Peq}}\right\rangle$ & & 3.57 & \\
\hline$\left\langle n_{\text {cvap }}\right\rangle$ & 8.93 & 6.68 & 4.65 \\
\hline$\left\langle n_{\mathrm{lol}}\right\rangle$ & 13.94 & 11.77 & 6.57 \\
\hline$\left\langle n_{5-10^{\circ}}\right\rangle$ & 0.094 & 0.086 & 0.04 \\
\hline$\left\langle n_{14(2-16)^{\circ}}\right\rangle$ & 1.012 & 1.176 & 0.44 \\
\hline$\left\langle p_{\text {INC }}\right\rangle$ & 2.45 & 0.74 & 1.63 \\
\hline$\left\langle p_{\mathrm{Peq}}\right\rangle$ & & 1.33 & \\
\hline$\left\langle p_{\text {evap }}\right\rangle$ & 0.74 & 0.23 & 0.45 \\
\hline$\left\langle p_{t o t}\right\rangle$ & 3.18 & 2.31 & 2.08 \\
\hline$\langle d\rangle$ & 0.28 & $0.60+0.04$ & 0.21 \\
\hline$\langle t\rangle$ & 0.17 & $0.21+0.02$ & 0.17 \\
\hline$\left\langle{ }^{3} \mathrm{He}\right\rangle$ & 0.08 & $0.06+0.00$ & 0.02 \\
\hline$\langle\alpha\rangle$ & 0.47 & $0.22+0.09$ & 0.32 \\
\hline$\left\langle n_{\text {tot }}(\right.$ free + composites $\left.)\right\rangle$ & 15.58 & 13.55 & 7.78 \\
\hline$\frac{\left.\left\langle n_{\text {tor }} \text { \{composites }\right)\right\rangle}{\left\langle n_{\text {tor }}(\text { Iree })\right\rangle}(\%)$ & 11.7 & 15.0 & 18.4 \\
\hline$\left\langle M_{\mathrm{enmp}}\right\rangle$ & 1.00 & 1.24 & 0.72 \\
\hline$\frac{\left\langle M_{\mathrm{uxmp} p}\right\rangle}{\left\langle N_{\text {free }}\right\rangle}(\%)$ & 5.9 & 8.8 & 8.3 \\
\hline
\end{tabular}

in Fig. 20, the resulting angle-integrated neutron spectrum, along with the results of our standard INC model. Clearly, the introduction of the pre-equilibrium step amounts to a reshuffling of the emitted particles from the so-called cascade ones to the pre-equilibrium ones. A little bit more detail is contained in Table 1 (which also shows results of the cascade+percolation model discussed below). One can see that the total number of expelled neutrons before evaporation is the same in both calculations, but it is $\sim 15 \%$ larger, after evaporation, in our standard model. The three step model produces less free (non-evaporative) neutrons at small angles and more at large angles than our standard model, a tendency which considerably worsens the comparison with experimental data. Also, the calculated mass spectrum (not shown) is less satisfactory in the three step model. In particular the so-called spallation peak is broader and the height of the fission peak is smaller than in our standard model (see Fig. 7). In conclusion, this first investigation shows that the three step model introduces unnecessary complications and arbitrariness (the choice of the stopping time) without improving the results. The only advantage is to allow (phenomenologically) the emission of composites in the early 
stages of the collision. Of course, a more thorough investigation is necessary before drawing general conclusions.

Let us comment about the predictions for average particle multiplicities. Unfortunately, only a few, indirect, measurements of these quantities have been done. From the neutron multiplicity distributions given in Ref. [12], one can estimate the average neutron multiplicity as being $\sim 6$ for the $\mathrm{p}(475 \mathrm{MeV})+\mathrm{Bi}$ system and $\sim 11$ for the $\mathrm{p}(2 \mathrm{GeV})+\mathrm{Au}$ system. These numbers should be taken with caution, as corrections for efficiencies had to be applied. They are probably lower bounds of the true average multiplicity. Recent measurements on the $\mathrm{p}(1.22 \mathrm{GeV})+\mathrm{Pb}$ system [41] yields an average neutron multiplicity of $\sim 15$. This value is rather an upper limit as the target used in this experiment is not really thin. One can see from Table 1, that the prediction of the INC+evaporation model is roughly consistent with this measurements. The value provided by INC+pre-cquilibrium+evaporation could not be ruled out. This discussion underlines the need for precise measurements of the neutron multiplicity. In Ref. [44], the experimental average $d / p$ ratio for the $\mathrm{n}(540 \mathrm{MeV})+\mathrm{Bi}$ reaction is quoted. It is equal to $\sim 0.1$. This is very close to the prediction of the INC+evaporation (the ratio is probably not varying much with the energy). On the contrary, the prediction of the INC+pre-equilibrium+evaporation model is close to 0.4 . However, we have to add that the experiment of Ref. [44] does not cover a large energy range. Finally, it has to be noticed that all the three models predict that a sizable fraction of neutrons is emitted inside composites.

\section{A cascade plus percolation approach}

To overcome the inability of the standard INC cascade to generate clusters of any size, we want to present here another version of the Liège cascade which allows for the break-up of the system under certain conditions. Actually this version was originally proposed in Ref. [42], for the case of heavy ion reactions. The idea is to use the standard INC model, neglecting the mean field. In order to avoid the spurious dispersion of the system, the Fermi motion of a target particle is introduced once it has collided with an active particle (here, an active particle is either the incident particle or a particle which has collided with the latter or with another active particle). At the end of the cascade (with $t_{\text {fin }}$ defined as explained in Ref. [8]), the spatial distribution of the nucleons is analyzed with a percolation procedure, described in Ref. [42]. In short, a minimum spanning tree [43] is constructed and links between nucleons with a length larger than a given minimum distance $d_{\text {cut }}$ are cut down. Fragments are defined by the remaining links. The kinetic energy of the clusters and their excitation energy are evaluated from the individual energy and momentum at the end of the cascade. In order to take account of the energy necessary to break the system, some corrections are necessary. For the free nucleons, their kinetic energy is reduced by the value $V_{0}$ of the depth of the neglected initial mean field potential. If the corrected value is negative, the nucleon is attached to the closest cluster. 


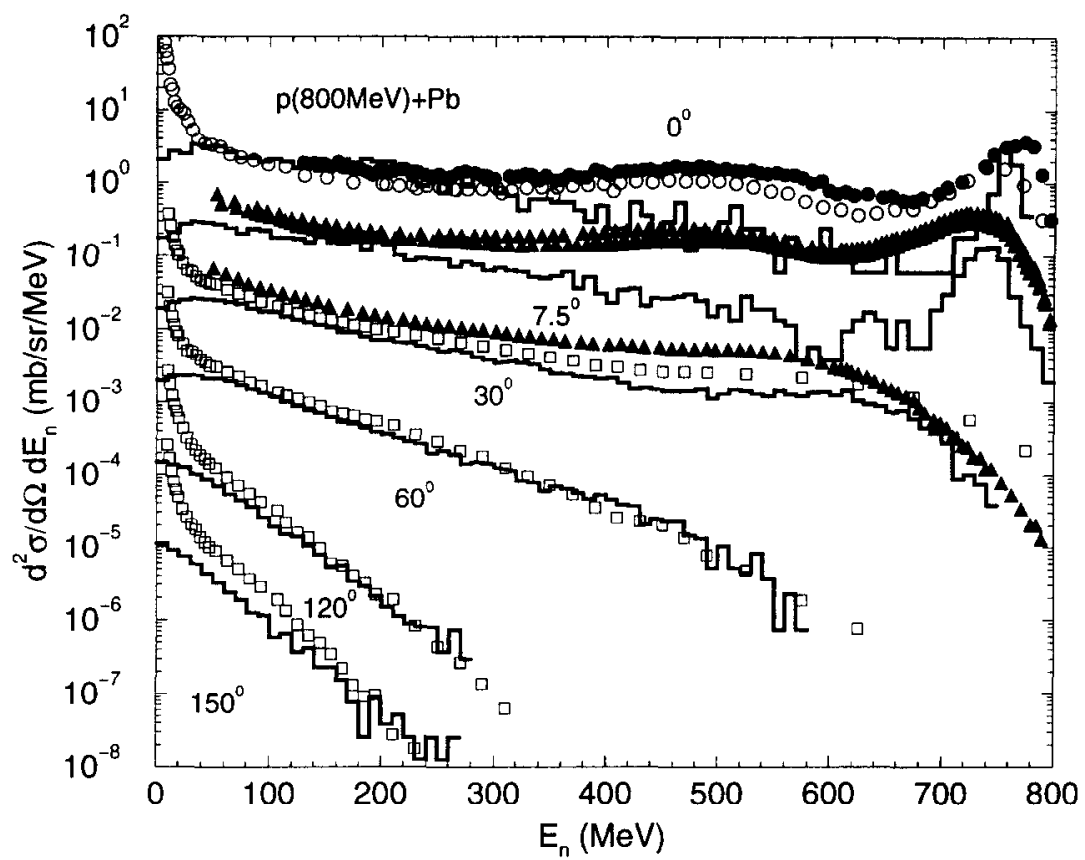

Fig. 21. Neutron double differential cross-sections in $\mathrm{p}(800 \mathrm{MeV})+\mathrm{Pb}$ reactions. Comparison between experiment (symbols) and the results of the INC+percolation model (histograms) described in Section 5 . Same convention as in Fig. 2.

We give in Fig. 21 the neutron spectra, calculated with this version of the cascade model, for the $\mathrm{p}(800 \mathrm{MeV})+\mathrm{Pb}$ system. The results are rather similar to the standard cascade (see Fig. 2), except for the very low neutron energy, especially at large angles. The reason for the difference comes presumably to the crude correction brought to the neutron kinetic energy. In Fig. 22, we display the deuteron spectra measured by the authors of Ref. [44] in the case of the $n(540 \mathrm{MeV})+\mathrm{Bi}$ system, along with the results of the cascade+percolation approach and the cascade+pre-equilibrium approach, presented in the preceding section. The agreement with the results is quite satisfactory.

The residue mass spectrum, obtained with the cascade+percolation model, is shown in Fig. 23, both before and after the evaporation step, for the $\mathrm{p}(1 \mathrm{GeV})+\mathrm{Au}$ system. The width of the spallation peak is substantially larger, in comparison with the standard model, both before and after evaporation. It seems however that a reasonable agreement with experimental data could be achieved if the clusters were moderately less excited. This would be obtained if part of the correction for the missing potential energy in the cascade was applied to the excitation energy of the fragments and not to the free neutrons only. On the other hand, the width of the spallation peak before evaporation can be diminished by using a larger value of $d_{\text {cut }}$. In other words, there is some room inside this model to improve the residue mass spectrum, but this requires a study of the optimisation of the parameters contained in this model. Let us stress the nicest feature of this model, namely its ability to generate intermediate mass fragments (intermediate 


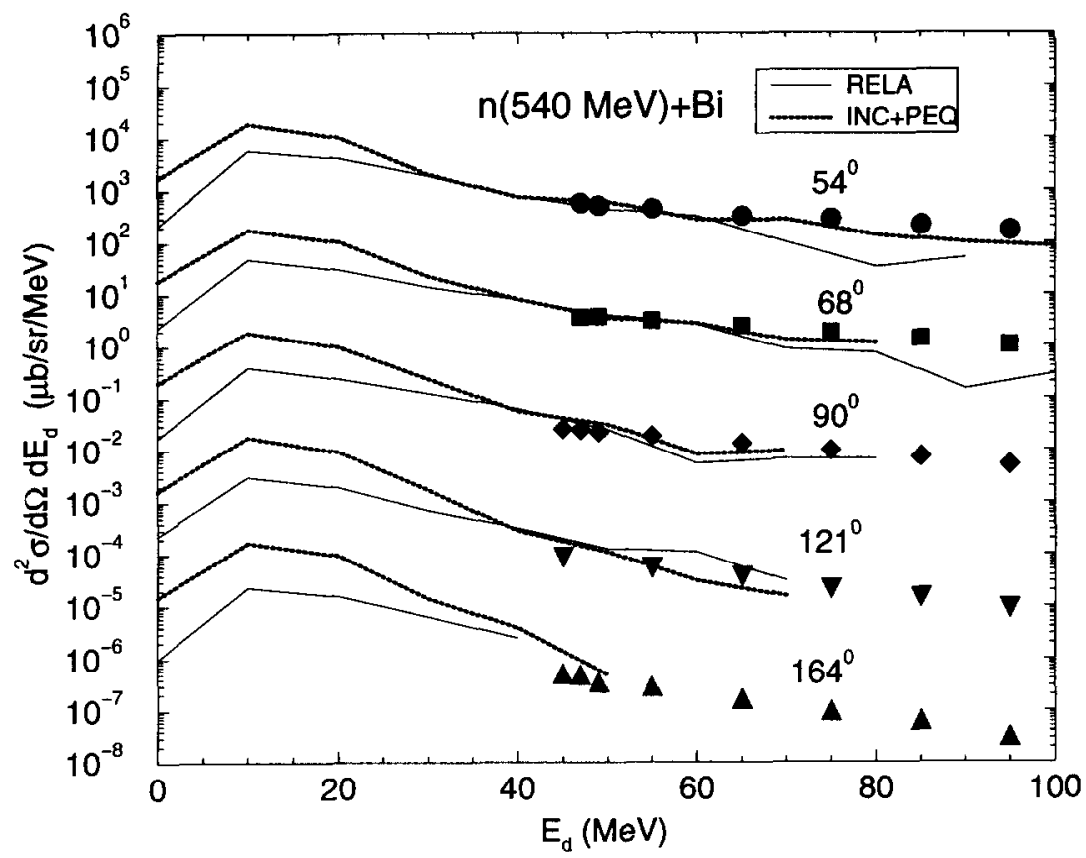

Fig. 22. Deuteron double differential cross-sections in $n(540 \mathrm{MeV})+\mathrm{Bi}$ reactions. Comparison between experiment [44] (black symbols), the results of the INC+pre-equilibrium model (dotted curves), described in Section 4, and those obtained by the INC+percolation model (referred to as RELA, full curves) described in Section 5. To avoid confusion, only the data and the curves relative to the smallest angle are plotted in absolute value. They are multiplied by $10^{-2}, 10^{-4}, \ldots$, for larger angles, in increasing order. See text for detail.

between the alpha particle mass and smallest typical fission fragment mass). The two other models investigated in this paper can hardly produce such fragments (and only in the evaporation step). The yield for intermediate mass fragments is small in reactions induced by protons of $\sim 1 \mathrm{GeV}$ or less, but it becomes more and more important for increasing incident energy [42]. In addition, this model can generate composites in the cascade stage (see Table 1).

\section{Discussion and conclusion}

In this paper, we have first demonstrated the generally good agreement obtained in the comparison of experimental data with the results of the standard Liège INC model, described in Section 2, for the two main quantities measured experimentally in nucleoninduced spallation reactions, namely neutron double differential cross-section and residue mass spectrum (see Figs. 2-4). When necessary, the INC model has been supplemented with the Dresner evaporation model [21], based on the Weisskopf-Ewing [45] theory and a phenomenological approach for the overall fission probability [46]. As for neutron production, the respective contributions of INC and evaporation can be separated, almost 


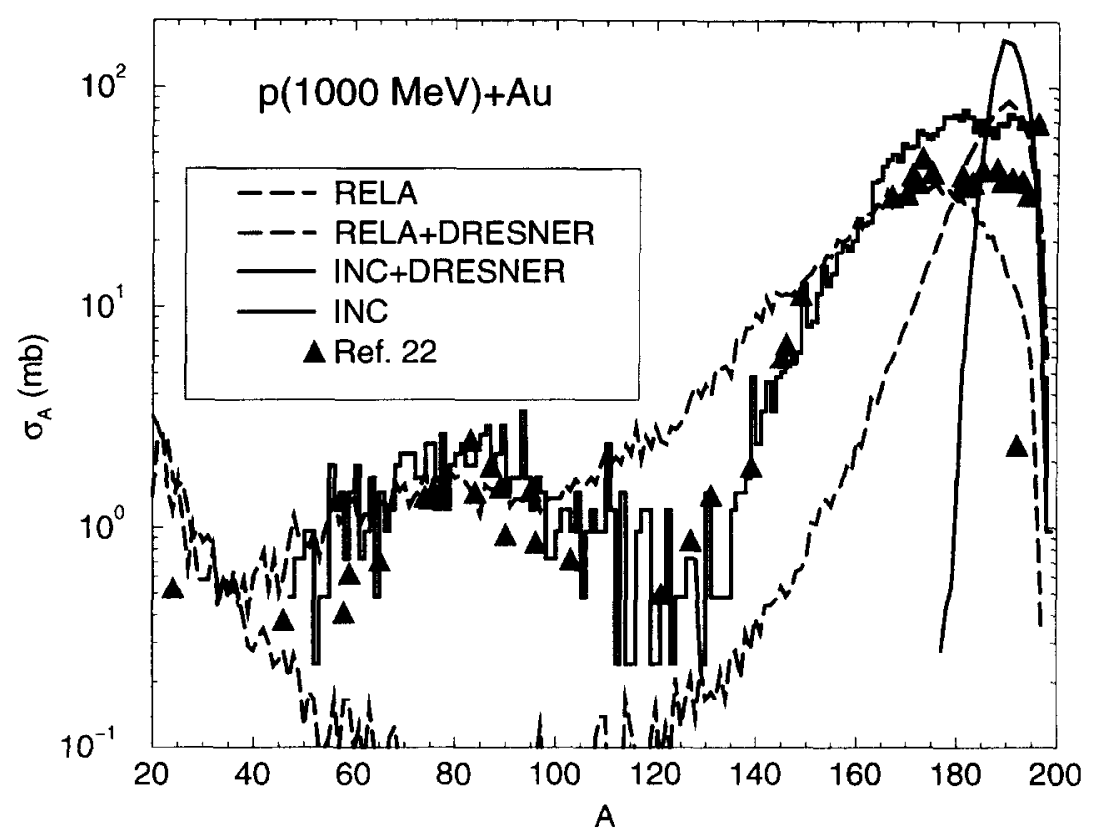

Fig. 23. Residue mass spectrum after $\mathrm{p}(\mathrm{I} \mathrm{GeV})+{ }^{197} \mathrm{Au}$ reactions. Data (symbols) are taken from Ref. [22] Theoretical results are obtained in an INC+evaporation model (full lines) and in the INC+percolation (referred to as RELA) +evaporation model (dashed lines), summing over $\sim 10^{5}$ events. In both cases, the evaporation model of Ref. [21] is used, and the residue mass spectrum before evaporation is also displayed.

by simply splitting the neutron energy domain into two parts. For energies larger than a few $\mathrm{MeV}$, the INC contribution is overwhelmingly dominant. The nice overall agreement obtained for neutron double differential cross-section is thus largely attributable to the INC model. We recall that the standard Liège INC model does not contain any free parameter. The basic ingredients are the nucleon-nucleon cross-sections (or their accurate parametrization, see Ref. [14] and Section 2) and known quantities describing the gross properties of the target (radius, Fermi momentum and mean field intensity). Even the stopping time of the cascade has been determined in a "self-consistent" manner, i.e. by the properties of the INC process itself, as we extensively explained in Sections 2 and 3 . On the other hand, for the residue mass spectrum, the respective contributions of the INC and evaporation (+fission) models are more entangled. The presence of a peak, centered on a mass value a few mass units below the target one, and not on the latter value, can be considered as a direct consequence of our INC model. The width of the peak is determined by both the INC and evaporation models. The presence of the fission bump is due to the evaporation (+fission) step, but its height also depends upon the excitation energy left in the remnant after the cascade stage.

The standard Liège INC model does not contain free parameters, as we just recalled, but rests on some simplifying assumptions, the most important of which is the one of a constant mean field. In the introduction, we give plausible arguments in support of this assumption. The wellfoundedness of this assumption was checked recently by the 
results of QMD calculations, supplemented by some statistical decay model [59]. We would like to stress that in the energy range investigated in this paper, our results are practically as good as those of the latter calculations. However, one has to keep in mind that our numerical program is much simpler and by more than an order of magnitude faster. It is thus more practical for particle transport study in macroscopic media.

The overall good agreement that we just discussed strongly indicates that the INC+evaporation model carries the right physics, namely the dominance of binary collisions, much as in free space, as long as the excitation energy is not too small, and of evaporation-like processes when the latter is small enough. One may worry about the fact that the mere addition of two processes, one involving individual degrees of freedom and the other dealing with properties of the whole system and including strong $A$-body correlations (embodied by the $a$-parameter of the evaporation model, usually far from its singleparticle, uncorrelated, value), can give a good description of the whole process. We think that this is due to the fact that the energy and particle flows in both models are however still largely correct when the actual physical situation involves mild (neither weak nor strong) correlations. In the INC model, when the excitation energy is becoming smaller and smaller, the Pauli principle and the constant rearrangement of the Fermi sea are hindering the emission of particles. As far as the outgoing energy and particle flows are concerned, they largely play the role of the interaction correlations. In the evaporation model, the evaporation pattern (number and energy of evaporated particles) is presumably more and more dominated by the value of the excitation energy (in comparison to the other parameters), when the latter is getting larger and larger. That is presumably the reason why, grossly speaking, there is no evident discontinuity between the output of the INC and evaporation steps. However, we want to stress that, even if the particle and energy flows are similar in the two models in the intermediate stage of the reaction processes, the time evolution of these flows may be quite different in the two models. To be more specific, the rate of emitted particles in the INC model at $t=t_{\text {fin }}$ may be quite different from the rate of nucleon emission in the evaporation model. For practical purposes, this is of no importance. In usual evaporation calculations, the absolute time rate does not enter into play, as only relative rates are used. This point is however not without theoretical interest and would deserve further investigations.

Although the gross features of the physics are correctly handled by our standard INC (+evaporation) model, fine details are probably missed, as systematic discrepancies are identified, as we explained in Section 2. Furthermore, these discrepancies seem to be too large, for using this model as a basic ingredient in particle transport studies in technological assemblies like the so-called spallation sources or the future acceleratorassisted subcritical systems [47,48]. For that reason, we investigated, in Section 3, several possible improvements of our standard model, still remaining in the framework of a quasi-classical picture, where particles are following well defined trajectories. Let us shortly discuss the role of the modifications in the comparison with experiment. Basically, our INC model is quite good in describing the spectra at large angles. For incident protons of $800 \mathrm{MeV}$, at small angles, our model is still quite good for a neutron energy less than say, 300-400 MeV. In the domain so defined, the discrepancy 
is generally small, always smaller than $50 \%$. In the $\mathrm{p}(800 \mathrm{MeV})+\mathrm{Pb}$ system, the discrepancy is larger and sometimes larger than a factor 2, for high energy neutrons ( $\gtrsim 400 \mathrm{MeV}$ ) emitted at very forward angles. This is particularly clear at $7.5^{\circ}$ (see Fig. 2), even if one considers the lowest value data. At lower incident energy, the discrepancy seems to survive, though attenuated, but then involves neutrons of lower energy. The domain where the discrepancy is larger than $50 \%$ is characterized by the dominance of the single-scattering contribution (this is illustrated by Figs. 2 and 4 in the $\mathrm{p}(800 \mathrm{MeV}) \mathrm{Pb}$ case). Let us stress that this corresponds to a small fraction (less than $6 \%$ ) of the total integrated cross-section.

The systematic character of this deficiency clearly establishes, in our opinion, the too weak relative importance of the single-scattering contribution. Either, the latter is underestimated in absolute value, or the double, triple, ... scattering contributions are overestimated. The latter processes indeed remove particles from the high-energy, forward angle domain. In all the modifications studied above, the only one which seems to increase the absolute value of the single-scattering component is the introduction of the diffuse surface (which, of course, is mandatory). The introduction of the hadronisation time, whatever its true physical meaning, seems to improve the results by reducing the relative importance of the multiple scattering contributions. Finally, it should not be forgotten that the single scattering contribution may be sensitive to the quantum corrections. For instance, in a strongly interacting fermion system, particles are no longer on their energy shell. This point would deserve detailed investigation.

Let us shortly discuss the results concerning the other modifications. The introduction of in-medium cross-sections poses an intriguing problem. Let us leave aside the inelastic cross-sections, for which in-medium cross-sections are not yet known with good confidence. On the contrary, it is widely believed that Brueckner theory is a good theoretical framework for evaluating in-medium effects on elastic scattering. As we showed in Section 3, adopting this approach does not modify the single-collision contribution. Introducing refraction does not help very much and even deteriorates the low energy spectra. One may wonder in fact, whether introducing the refraction picture is not pushing the similarity with wave optics too far, especially in incoherent processes as the ones we are facing here. The improved handling of the Pauli principle does not seem of real importance. The collision mode, except for the hadronisation time, does not play any significant role, as we indicated in Section 3. If the stopping time is considered as a potential improvement, changes of the latter within reasonable values does not change the neutron spectra but are more important for the gross properties of the residue mass spectrum, because it mainly controls the position of the system in the $\left(A, E^{*}\right)$ plane at the end of the cascade. The latter being not really correlated to the detail of the distribution of the emitted neutrons, we do not expect the residue mass spectrum to be very sensitive to the other modifications described above.

Other observables may be of importance, at least in the scope of technological applications. Among these, let us mention the multiplicity and distribution of composite particles, even if it is experimentally established that their total multiplicity is of the order of $10 \%$ of the total particle multiplicity, at the most, in the energy range under 
consideration [44]. In our standard+evaporation model, the composites come from the evaporation stage alone. We have investigated in Sections 4 and 5, two models in which the evaporation is not the single source. In one case, the other source comes from a pre-equilibrium stage. In the other, it comes from a percolation procedure, which is supposed to simulate the many-body correlations built by the cascade process. Both give a reasonable value of the deuteron production measured in Ref. [44]. We also investigate the pre-equilibrium stage as a practical mean to pass smoothly from the hard cascade stage to the soft evaporation stage. We draw the attention to the similarity of the pre-equilibrium model and the INC model and thus to the redundancy of the former. We showed that the introduction of this intermediate step is confusing and not really necessary.

In conclusion, we have shown that the standard Liège INC+evaporation model provides an already good agreement with experimental data in nuclcon-induced reactions in the $\mathrm{GeV}$ range. We have identified its (small) deficiency and proposed a way to correct it. The systematic use of the so improved model and its applications to higher energy (and other projectiles) will be described in further publications. We have also shown that the introduction of an intermediate step is not really required. Finally, let us mention that the improvements of the third category mentioned in the introduction should be investigated. In particular, the apparently very small place left for quantum corrections should be understood.

\section{Acknowledgements}

We would like to thank Drs S. Leray and G. Cohen-Tannoudji for helpful discussions. We are very grateful to the authors of Ref. [18] for providing us with part of their data prior to publication and to Dr S.G. Mashnik for allowing to use his pre-equilibrium numerical code.

\section{References}

[1] S. Nagamyia and M. Gyulassy, Adv. Nucl. Phys. 13 (1982) 201.

[2] D.L'Hôte and J. Cugnon, in Relativistic Heavy Ion Collisions, ed. L.P. Csernaï and D. Strottman, Int. Rev. Nucl. Phys. 5 (World Scientific, Singapore, 1991) p. 37.

[3] M. Soyeur, H. Flocard, B. Tamain and M. Porneuf, Nuclear Matter and Heavy lon Collisions, NATO ASI Series B Physics, Vol. 205 (Plenum Press, New York-London, 1989).

[4] J. Aichelin, Phys. Rep. C 202 ( 1991) 233.

[5] J. Cugnon, Nucl. Phys. A 462 (1987) 751.

[6] J. Cugnon and J. Vandermeulen, Ann. Phys.(Fr) 14 (1989) 49.

[7] Ye. Golubeva, A.S. Iljinov, A.S. Botvina and N.M. Sobolevsky, Nucl. Phys. A 483 (1988) 539.

[8] L. Pienkowski et al., Acta Physica Polonica B 25 (1994) 737.

[9] C.D. Bowman et al., Nucl. Instr. and Meth. A 320 (1992) 336.

[10] S. Andriamonje et al., Phys. Lett. B 348 (1995) 697.

[11] J. Cugnon and M.C. Lemaire, Nucl. Phys. A 489 (1988) 781.

[12] L. Pienkowski et al., Phys. Lett. B 336 (1994) 147.

[13] J. Cugnon, T. Mizutani and J. Vandermeulen, Nucl. Phys. A 352 (1981) 505. 
[14] J. Cugnon, D. L'Hôte and J. Vandermeulen, Nucl. Instr. and Meth. B 111 (1996) 215.

[15] G.F. Chew, Phys. Rev.112 (1958) 1380.

[16] L.C. Northcliffe et al., Phys. Rev. C 47 (1993) 36.

117 H.W. Bertini, Phys. Kev. 131 (1963) 1801.

[18] E. Martinez et al., to be submitted to Nucl. Instr. and Meth..

[19] J. Cugnon, S. Leray, E. Martinez, Y. Patin and S. Vuillier, submitted to Phys. Rev. C

[20] F.M. Waterman et al., Phys. Rev. C 8 (1973) 2419.

[21] L. Dresner, Oak Ridge report ORNL-TM-196 (1962).

[22] S.B. Kaufman and E.P. Steinberg, Phys. Rev. C 22 (1980) 167.

[23| J. Cugnon, A. Lejeune and P. Grangé, Phys. Rev. C 35 (1987) 861.

[24] K.A. Brueckner, Phys. Rev. 97 (1955) 1353.

[25] W. Botermans and R. Malfliet, Phys. Lett. B 215 (1988) 671.

|26] R. Malfliet, Nucl. Phys. A 545 (1992) 3c.

[27] C.Q. Li and R. Machleidt, Phys. Rev. C 48 (1993) 1702.

[28] C.Q. Li and R. Machleidt, Phys. Rev. C 49 (1994) 566.

[29] J. Cugnon and R. Sartor, to be published..

[30] H. Takada, J. Nucl. Sci. Tech. 33 (1996) 275.

[31] B. ter Haar and R. Malfliet, Phys. Rev. C 36 (1987) 1611.

[32] S. Chiba, O. Iwamoto, T. Fukahori, K. Niita, T. Maruyama, T. Maruyama and A. Iwamoto, Phys. Rev. C 54 (1996) 285.

[33] P.G. Reinhard and E. Suraud, Z. Phys. A 355 (1996) 339.

[34] M. Gyulassy, K. Frankel, E.A. Remler, Nucl. Phys. A 402 (1983) 596.

[35] F. Karsch, M.T. Melir and H. Satz, Z. Phys. C 37 (1988) 617.

[36] J.P. Blaizot and J.Y. Ollitrault, Phys. Lett. B 217 (1991) 386.

[37] P. Danielewiecz and S. Pratt, Phys. Rev. C 53 (1996) 249.

[38] M. Blann, Ann. Rev. Nucl. Sci. 25 (1975) 123.

[39] E. Gadioli and P.E. Hodgson, Pre-Equilibrium Nuclear Reactions (Clarendon, Oxford, 1992).

[40] S.G. Mashnik, K.K. Gudima, V.D. Toneev, Nucl. Phys. A 401 (1983) 329.

141 | D. Hilscher, F. Goldenbaum, U. Jahnke, L. Pienkowski, J. Galin, B. Lott and B. Quednau, contribution to the Int. Workshop on Nuclear Methods for Transmutation of Nuclear Waste: Problems, Perspectives, Cooperative Research, Dubna, May 1996, HMI preprint (1996).

[42] J. Cugnon and C. Volant, Z. Phys. A 334 (1989) 435.

[43] J. Dorfan, Z. Phys. C 7 (1981) 349.

[44] J. Franz et al., Nucl. Phys. A 510 (1990) 774.

[45] V.F. Weisskopf and D.H. Ewing , Phys. Rev. 57 (1940) 472,935.

[46] F. Atchison, Proc. Meeting on Targets for Neutron Spallation Sources, Jülich Conf. 34 (1980) 17.

147] International Code Comparison for Intermediate Energy Nuclear Data, OECD/NEA (1994).

|48| M. Salvatorès, private communication (1996).

[49| M.L. Ewans et al., Phys. Rev. C 26 (1982) 2525.

[50] Y. Terrien et al., Phys. Rev. Lett. 59 (1987) 1534.

[51] S. Stamer et al., Phys. Rev. C 47 (1993) 1647.

[52] W.B. Amian et al., Nucl. Sci. Eng. 112 (1992) 78.

[53| M.M. Meier et al., Rad. Eff. 96 (1986) 73.

[54] W. Scobel et al., Phys. Rev. C 41 (1990) 2010.

$155 \mid$ B.E. Bonner et al., Phys. Rev. C 18 (1978) 1418.

[56] W.B. Amian et al., Nucl. Sci. Eng. 110 (1992) 289.

[57| W.B. Amian et al., Nucl. Sci. Eng. 115 (1993) 1.

[58] X. Blanchard et al., 2nd Int. Conf. on Accelerator-Driven Transmutation Technologies and Applications, Kalmar, Sweden, June 3-7 (1996)

[59] K. Niita, S. Chiba, T. Maruyama, T. Maruyama, H. Takada, T. Fukahori, Y. Nakahara and A. Iwamoto, Phys. Rev. C 52 (1995) 2620. 